\title{
Stability of Solitary Waves of a Generalized Ostrovsky Equation
}

\author{
Steve Levandosky* and Yue Liu ${ }^{\dagger}$
}

\begin{abstract}
Considered herein is the stability problem of solitary wave solutions of a generalized Ostrovsky equation, which is a modification of the Korteweg-de Vries equation widely used to describe the effect of rotation on surface and internal solitary waves or capillary waves.
\end{abstract}

\section{Introduction}

The nonlinear dispersive equation

$$
\left(u_{t}-\beta u_{x x x}+\left(u^{2}\right)_{x}\right)_{x}=\gamma u, \quad x \in \mathbf{R},
$$

was derived by Ostrovsky [20] in dimensionless space-time variables $(x, t)$ as a model for the unidirectional propagation of weakly nonlinear long surface and internal waves of small amplitude in a rotating fluid. The liquid is assumed to be incompressible and inviscid. Here $u(t, x)$ represents the free surface of the liquid and the parameter $\gamma>0$ measures the effect of rotation. The parameter $\beta$ determines the type of dispersion, namely, $\beta<0$ (negative dispersion) for surface and internal waves in the ocean or surface waves in a shallow channel with an uneven bottom and $\beta>0$ (positive dispersion) for capillary waves on the surface of liquid or for oblique magneto-acoustic waves in plasma. See Benilov [2], Galkin, Stepanyants and Gilman [7] and Gilman, Grimshaw and Stepanyants [8].

Considered herein is the generalization of the Ostrovsky equation

$$
\left(u_{t}-\beta u_{x x x}+f(u)_{x}\right)_{x}=\gamma u, \quad x \in \mathbf{R}
$$

where $f$ is a $C^{2}$ function which is homogeneous of degree $p \geq 2$, in the sense that it satisfies $s f^{\prime}(s)=p f(s)$. This includes for instance nonlinearities of the form $f(u)= \pm|u|^{p}$ and $\pm|u|^{p-1} u$. Certain equations of this class have a direct relation to physical systems. In particular, when $p=3$, equation (1.2) describes the propagation of internal waves of even

\footnotetext{
*Mathematics and Computer Science Department, College of the Holy Cross, Worcester, MA 01610, spl@mathcs.holycross.edu

${ }^{\dagger}$ Department of Mathematics, The University of Texas at Arlington, Arlington, TX 76019, yliu@uta.edu
} 
modes, which possess a cubic nonlinearity, in the ocean. See Galkin and Stepanyants [7], Leonov [13] and Shrira [22, 23].

In this paper, we investigate the stability of solitary wave solutions of (1.2). Using variational methods we prove the existence of solitary waves (Theorem 2.1). Solitary waves thus obtained are called ground states and the set of all ground states is denoted by $G(\beta, c, \gamma)$. The variational characterization of the ground states permits us to consider the limiting behavior of the solitary waves as the rotation parameter $\gamma$ vanishes, and we show that the ground state solitary waves converge to solitary waves of the KdV equation (Theorem 2.5).

The stability analysis makes use of the conserved quantities

$$
E(u)=\int_{\mathbf{R}} \frac{\beta}{2} u_{x}^{2}+\frac{\gamma}{2}\left|D_{x}^{-1} u\right|^{2}+F(u) d x
$$

and

$$
V(u)=\frac{1}{2} \int_{\mathbf{R}} u^{2} d x
$$

where $F^{\prime}=f$ and $F(0)=0$ and the operator $D_{x}^{-1}$ is defined via the Fourier transform as

$$
\widehat{D_{x}^{-1} f}=(-i \xi)^{-1} \hat{f}(\xi) \text {. }
$$

It was shown by Liu and Varlamov [18] that the classical Ostrovsky equation (1.1) is wellposed in the space

$$
X_{s}=\left\{f \in H^{s}(\mathbf{R}) \mid D_{x}^{-1} f \in H^{s}(\mathbf{R})\right\}
$$

with norm

$$
\|f\|_{X_{s}}=\|f\|_{s}+\left\|D_{x}^{-1} f\right\|_{s}
$$

for $s>3 / 2$. The methods therin also imply the same result for the generalized Ostrovsky equation (1.2). We therefore make the following definition.

Definition 1.1. A set $S \subset X$ is $X$-stable with respect to equation (1.2) if for any $\epsilon>0$ there exists $\delta>0$ such that for any $u_{0} \in X \cap X_{s}, s>3 / 2$, with

$$
\inf _{v \in S}\left\|u_{0}-v\right\|_{X}<\delta
$$

the solution $u(t)$ of $(1.2)$ with initial value $u_{0}$ can be extended to a solution in $C([0, \infty), X \cap$ $\left.X_{s}\right)$ and satisfies

$$
\inf _{v \in S}\|u(t)-v\|_{X}<\epsilon
$$

for all $t \geq 0$. Otherwise we say that $S$ is $X$-unstable.

Our main results apply to the set $G(\beta, c, \gamma)$, defined by (2.8). For each $y \in \mathbf{R}$ we define the translation operator by $\tau_{y} v=v(\cdot+y)$. Given a ground state $\varphi$ in $G(\beta, c, \gamma)$, the orbit of $\varphi$ is the set $\mathcal{O}_{\varphi}=\left\{\tau_{y} \varphi \mid y \in \mathbf{R}\right\}$. We show, in Theorems 3.1 and 4.2 that the function $d$ defined by (3.1) determines the stability or instability of the solitary waves in the sense that if $d^{\prime \prime}(c)>0$, then $G(\beta, c, \gamma)$ is $X_{1}$-stable and if $d^{\prime \prime}(c)<0$, then $\mathcal{O}_{\varphi}$ is $X_{1}$-unstable. Although these results are not quite complementary, the only difference is due to the possible nonuniqueness of ground states up to translation. That is, if ground states are unique up to translation, then $G(\beta, c, \gamma)=\mathcal{O}_{\varphi}$. 
One difficulty in applying these results is the fact that an explicit formula for $d$ is not available. It is also not known if $d(c)$ is twice differentiable. To remedy this, we also prove a second result, Theorem 4.3, which provides sufficient conditions for instability directly in terms of the parameters $\beta, c, \gamma$ and $p$. The result is based on the work of Goncalves Rebeiro [10]. Another approach to dealing with the lack of information about $d(c)$ is to compute it numerically. We conclude the paper with some numerical calculations of $d^{\prime \prime}$ which approximately determine regions of stability and instability in terms of the parameters.

Notation. The norm in the classical Sobolev spaces $H^{s}(\mathbf{R})$ will be written $\|\cdot\|_{s}$. For $1 \leq q \leq \infty$, the norm in $L^{q}(\mathbf{R})$ will be written $|\cdot|_{q}$.

\section{Solitary Waves}

Solitary-wave solutions of the form $u(x, t)=\varphi(x-c t)$ satisfy the stationary equation

$$
\beta \varphi_{x x}+c \varphi+\gamma D_{x}^{-2} \varphi=f(\varphi)
$$

We will prove existence of solitary waves in the space $X_{1}$ by considering the following variational problem. Define the functionals

$$
I(u)=I(u ; \beta, c, \gamma)=\int_{\mathbf{R}} \beta u_{x}^{2}-c u^{2}+\gamma\left(D_{x}^{-1} u\right)^{2} d x
$$

and

$$
K(u)=-(p+1) \int_{\mathbf{R}} F(u) d x,
$$

where $F$ satisfies $F^{\prime}=f$ and $F(0)=0$. Then if $\psi \in X_{1}$ achieves the minimum

$$
M_{\lambda}=\inf \left\{I(u) \mid u \in X_{1}, K(u)=\lambda\right\}
$$

for some $\lambda>0$, then there exists a Lagrange multiplier $\mu$ such that

$$
\beta \psi_{x x}+c \psi+\gamma D_{x}^{-2} \psi=\mu f(\psi) .
$$

Hence $\varphi=\mu^{\frac{1}{p-1}} \psi$ satisfies (2.1). We call such solutions ground state solutions and denote the set of all ground state solutions by $G(\beta, c, \gamma)$. By the homogeneity of $I$ and $K$, ground states also achieve the minimum

$$
m=m(\beta, c, \gamma)=\inf _{\substack{u \in X_{1} \\ K(u)>0}} \frac{I(u)}{(K(u))^{\frac{2}{p+1}}},
$$

and it follows that

$$
M_{\lambda}=m \lambda^{\frac{2}{p+1}} .
$$

We next note that the properties $s f^{\prime}(s)=p f(s)$ and $F^{\prime}=f$ imply that $s f(s)=(p+1) F(s)$, so that

$$
K(u)=-\int_{\mathbf{R}} u f(u) d x
$$


and therefore multiplying (2.1) by $\varphi$ and integrating yields $I(\varphi)=K(\varphi)$. Thus we may characterize the set of ground-state solutions $G(\beta, c, \gamma)$ as

$$
G(\beta, c, \gamma)=\left\{\varphi \in X_{1} \mid K(\varphi)=I(\varphi ; \beta, c, \gamma)=(m(\beta, c, \gamma))^{\frac{p+1}{p-1}}\right\}
$$

We now seek to prove that this set is non-empty. We say that a sequence $\psi_{k}$ is a minimizing sequence if for some $\lambda>0$,

$$
\lim _{k \rightarrow \infty} K\left(\psi_{k}\right)=\lambda \quad \text { and } \quad \lim _{k \rightarrow \infty} I\left(\psi_{k}\right)=M_{\lambda}
$$

Theorem 2.1. Let $\beta>0, \gamma>0$ and $c<2 \sqrt{\beta \gamma}$. Let $\psi_{k}$ be a minimizing sequence for some $\lambda>0$. Then there exists a subsequence (renamed $\psi_{k}$ ), scalars $y_{k} \in \mathbf{R}$ and $\psi \in X_{1}$ such that $\psi_{k}\left(\cdot+y_{k}\right) \rightarrow \psi$ in $X_{1}$. The function $\psi$ achieves the minimum $I(\psi)=M_{\lambda}$ subject to the constraint $K(\psi)=\lambda$.

Proof. The result is an application of the Concentration Compactness Lemma of Lions [16]. We outline the proof here. First observe that by equation (2.7), the strict subadditivity condition

$$
M_{\alpha}+M_{\lambda-\alpha}>M_{\lambda}
$$

holds for any $\alpha \in(0, \lambda)$. Next, since $\beta>0, \gamma>0$ and $c<2 \sqrt{\beta \gamma}$, the functional $I$ satisfies the coercivity condition

$$
I(u) \geq A \int_{\mathbf{R}} u_{x}^{2}+\left(D_{x}^{-1} u\right)^{2} d x=A\|u\|_{X_{1}}^{2}
$$

where

$$
A=\left\{\begin{array}{ccc}
\frac{4 \beta \gamma-c^{2}}{2\left(\beta+\gamma+\sqrt{\left.(\beta-\gamma)^{2}+c^{2}\right)}\right.} & \text { for } & 0<c<2 \sqrt{\beta \gamma} \\
\min \{\beta, \gamma\} & \text { for } & c \leq 0
\end{array}\right\}>0 .
$$

It is also clear that $I(u) \leq C\|u\|_{X_{1}}^{2}$ for some constant $C$, so $I(u)^{1 / 2}$ is equivalent to the norm on $X_{1}$. Now let $\psi_{k}$ be a minimizing sequence. Then by coercivity of $I$, the sequence $\psi_{k}$ is bounded in $X_{1}$, so if we define

$$
\rho_{k}=\left|D_{x} \psi_{k}\right|^{2}+\left|D_{x}^{-1} \psi_{k}\right|^{2}
$$

then after extracting a subsequence, we may assume

$$
\lim _{k \rightarrow \infty} \int_{\mathbf{R}} \rho_{k} d x=L>0
$$

We may assume further after normalizing that $\int_{\mathbf{R}} \rho_{k} d x=L$ for all $k$. By the Concentration Compactness Lemma, a further subsequence $\rho_{k}$ satisfies one of the following three conditions.

- Vanishing: For every $R>0, \lim _{k \rightarrow \infty} \sup _{y \in \mathbf{R}} \int_{B(y, R)} \rho_{k} d x=0$. 
- Dichotomy: There exists some $l \in(0, L)$ such that for any $\epsilon>0$ there exist $R>0$ and $R_{k} \rightarrow \infty, y_{k} \in \mathbf{R}$ and $k_{0}$ such that

$$
\left|\int_{B\left(y_{k}, R\right)} \rho_{k} d x-l\right|<\epsilon \text { and }\left|\int_{R<\left|x-y_{k}\right|<R_{k}} \rho_{k} d x\right|<\epsilon
$$

for $k \geq k_{0}$.

- Compactness: There exists $y_{k} \in \mathbf{R}$ such that for any $\epsilon>0$ there exists $R(\epsilon)$ such that

$$
\int_{B\left(y_{k}, R(\epsilon)\right)} \rho_{k} d x \geq \int_{\mathbf{R}} \rho_{k} d x-\epsilon
$$

for all $k$.

In the same manner as in [14], it follows from the coercivity of $I$, the Sobolev inequality and the subadditivity condition (2.9) that both vanishing and dichotomy may be ruled out, and therefore the sequence $\rho_{k}$ is compact. Now set $\varphi_{k}(x)=\psi_{k}\left(x+y_{k}\right)$. Since $\varphi_{k}$ is bounded in $X_{1}$, a subsequence $\varphi_{k}$ converges weakly to some $\psi \in X_{1}$, and by the weak lower semicontinuity of $I$ over $X_{1}$, we have

$$
I(\psi) \leq \lim _{k \rightarrow \infty} I\left(\varphi_{k}\right)=M_{\lambda} .
$$

Furthermore, weak convergence in $X_{1}$, compactness of $\rho_{k}$ and the Sobolev inequality imply strong convergence of $\varphi_{k}$ to $\psi$ in $L^{p+1}$. Therefore

$$
K(\psi)=\lim _{k \rightarrow \infty} K\left(\varphi_{k}\right)=\lambda,
$$

so $I(\psi) \geq M_{\lambda}$. Together with the inequality above, this implies $I(\psi)=M_{\lambda}$, so $\psi$ is a minimizer of $I$ subject to the constraint $K(\varphi)=\lambda$. Finally, since $I$ is equivalent to the norm on $X_{1}, \phi_{k} \rightarrow \psi$ and $I\left(\phi_{k}\right) \rightarrow I(\psi)$, it follows that $\phi_{k}$ converges strongly to $\psi$ in $X_{1}$.

At this time it is unknown whether or not the ground states are unique up to translation. Uniqueness would imply that if $\varphi \in G(\beta, c, \gamma)$ is any ground state, then $G(\beta, c, \gamma)=\mathcal{O}_{\varphi}$, in which case the stability and instability theorems (Theorems 3.1 and 4.2 ) are complementary. We suspect that the ground states are unique, at least in the case $c<-2 \sqrt{\beta \gamma}$, when the ground states have non-oscillatory tails.

The function $m(\beta, c, \gamma)$ defined above plays an important role in our later results, so we now will investigate some of its properties. The first is a simple scaling identity.

Lemma 2.2. Let $\beta>0, \gamma>0$ and $c<2 \sqrt{\beta \gamma}$. For any $r>0$ and $s>0$ we have

$$
m\left(r s^{2} \beta, r c, r s^{-2} \gamma\right)=r s^{\frac{p-1}{p+1}} m(\beta, c, \gamma)
$$

Proof. Let $u \in X_{1}$ with $K(u) \neq 0$. For any $r>0$ we have

$$
I(u ; r \beta, r c, r \gamma)=r I(u ; \beta, c, \gamma),
$$


so $m(r \beta, r c, r \gamma)=r m(\beta, c, \gamma)$. Next let $v(x)=u(s x)$ for $s>0$. Then

$$
I(v ; \beta, c, \gamma)=\frac{1}{s} I\left(u ; s^{2} \beta, c, s^{-2} \gamma\right) \quad K(v)=\frac{1}{s} K(u)
$$

SO

and consequently

$$
\frac{I(v ; \beta, c, \gamma)}{K(v)^{\frac{2}{p+1}}}=s^{\frac{1-p}{p+1}} \frac{I\left(u ; s^{2} \beta, c, s^{-2} \gamma\right)}{K(u)^{\frac{2}{p+1}}}
$$

$$
m\left(s^{2} \beta, c, s^{-2} \gamma\right)=s^{\frac{p-1}{p+1}} m(\beta, c, \gamma)
$$

Next, we show that $m$ is continuous and monotone in each of its variables.

Lemma 2.3. The function $m$ is continuous on the domain $\beta>0, \gamma>0, c<2 \sqrt{\gamma \beta}$. Furthermore, $m$ is strictly increasing in $\gamma$ and $\beta$ and strictly decreasing in $c$.

Proof. First, fix $\beta>0$ and $\gamma>0$ and consider $c_{1}<c_{2}<2 \sqrt{\beta \gamma}$. Let $\varphi_{c_{1}}$ and $\varphi_{c_{2}}$ be ground states with $c=c_{1}$ and $c=c_{2}$, respectively. Then

$$
\begin{aligned}
m\left(\beta, c_{2}, \gamma\right) & \leq \frac{I\left(\varphi_{c_{1}} ; \beta, c_{2}, \gamma\right)}{K\left(\varphi_{c_{1}}\right)^{\frac{2}{p+1}}} \\
& =\frac{I\left(\varphi_{c_{1}} ; \beta, c_{1}, \gamma\right)+\left(c_{1}-c_{2}\right) \int \varphi_{c_{1}}^{2} d x}{K\left(\varphi_{c_{1}}\right)^{\frac{2}{p+1}}} \\
& =\frac{I\left(\varphi_{c_{1}} ; \beta, c_{1}, \gamma\right)}{K\left(\varphi_{c_{1}}\right)^{\frac{2}{p+1}}}+\left(c_{1}-c_{2}\right) \frac{\int \varphi_{c_{1}}^{2} d x}{K\left(\varphi_{c_{1}}\right)^{\frac{2}{p+1}}} \\
& =m\left(\beta, c_{1}, \gamma\right)+\left(c_{1}-c_{2}\right) \frac{\int \varphi_{c_{1}}^{2} d x}{K\left(\varphi_{c_{1}}\right)^{\frac{2}{p+1}}} \\
& <m\left(\beta, c_{1}, \gamma\right),
\end{aligned}
$$

so $m$ is strictly decreasing in $c$. On the other hand,

$$
\begin{aligned}
m\left(\beta, c_{1}, \gamma\right) & \leq \frac{I\left(\varphi_{c_{2}} ; \beta, c_{1}, \gamma\right)}{K\left(\varphi_{c_{2}}\right)^{\frac{2}{p+1}}} \\
& =\frac{I\left(\varphi_{c_{2}} ; \beta, c_{2}, \gamma\right)+\left(c_{2}-c_{1}\right) \int \varphi_{c_{2}}^{2} d x}{K\left(\varphi_{c_{2}}\right)^{\frac{2}{p+1}}} \\
& =m\left(\beta, c_{2}, \gamma\right)+\left(c_{2}-c_{1}\right) \frac{\int \varphi_{c_{2}}^{2} d x}{K\left(\varphi_{c_{2}}\right)^{\frac{2}{p+1}}}
\end{aligned}
$$

SO

$$
0 \leq m\left(\beta, c_{1}, \gamma\right)-m\left(\beta, c_{2}, \gamma\right) \leq\left(c_{2}-c_{1}\right) \frac{\int \varphi_{c_{2}}^{2} d x}{K\left(\varphi_{c_{2}}\right)^{\frac{2}{p+1}}}
$$

Now since

$$
I\left(\varphi_{c_{2}} ; \beta, c_{2}, \gamma\right) \geq A \int \varphi_{c_{2}}^{2} d x
$$


where $A$ is defined by (2.10), it follows that

$$
\left|m\left(\beta, c_{2}, \gamma\right)-m\left(\beta, c_{1}, \gamma\right)\right| \leq A^{-1} m\left(\beta, c_{2}, \gamma\right)\left(c_{2}-c_{1}\right)
$$

so $m$ is locally Lipschitz continuous in $c$. By similar reasoning it follows that $m$ is increasing and locally Lipschitz in $\beta$ and $\gamma$.

We now consider the effect of letting the rotation parameter $\gamma$ approach zero. Formally, this results in the generalized $\mathrm{KdV}$ equation

$$
\beta \varphi_{x x}+c \varphi=f(\varphi)
$$

For $c<0$, ground state solutions of (2.11) achieve the minimum

$$
m(\beta, c, 0)=\inf _{\substack{u \in H^{1} \\ K(u)>0}} \frac{I(u ; \beta, c, 0)}{K(u)^{\frac{2}{p+1}}}
$$

where

$$
I(u ; \beta, c, 0)=\int \beta u_{x}^{2}-c u^{2} d x,
$$

and $K$ is defined as before by equation (2.3). Thus the set of ground states may be characterized as

$$
G(\beta, c, \gamma)=\left\{\varphi \in H^{1} \mid K(\varphi)=I(\varphi ; \beta, c, 0)=(m(\beta, c, 0))^{\frac{p+1}{p-1}}\right\} .
$$

Moreover, it is well-known that the ground states are unique up to translation, so that $G(\beta, c, 0)=\left\{\varphi_{0}(\cdot-y) \mid y \in \mathbf{R}\right\}$ for some $\varphi_{0}$. For example, in the case of the nonlinearity $f(u)=(-u)^{p}$ where $p \geq 2$ is an integer, we have the explicit formula

$$
\varphi_{0}(x)=-\left(\frac{-c(p+1)}{2}\right)^{\frac{1}{p-1}} \operatorname{sech}^{\frac{2}{p-1}}\left(\frac{p-1}{2} \sqrt{\frac{-c}{\beta}} x\right) .
$$

Therefore the analogue of Theorem 2.1 takes the following form.

Theorem 2.4. Let $\beta>0$, and $c<0$. Let $\psi_{k}$ be a sequence in $H^{1}$ such that

$$
\lim _{k \rightarrow \infty} I\left(\psi_{k} ; \beta, c, 0\right)=\lim _{k \rightarrow \infty} K\left(\psi_{k}\right)=(m(\beta, c, 0))^{\frac{p+1}{p-1}} .
$$

Then there exists a subsequence (renamed $\psi_{k}$ ), scalars $y_{k} \in \mathbf{R}$ such that $\psi_{k}\left(\cdot+y_{k}\right) \rightarrow \varphi_{0}$ in $H^{1}$.

Theorem 2.5. Fix $\beta>0$, and $c<0$ and consider any sequence $\gamma_{k} \rightarrow 0^{+}$. Denote by $\varphi_{k}$ any element of $G\left(\beta, c, \gamma_{k}\right)$. Then there exists a subsequence (renamed $\gamma_{k}$ ) and translations $y_{k}$ so that

$$
\varphi_{k}\left(\cdot+y_{k}\right) \rightarrow \varphi_{0}
$$

in $H^{1}$, as $\gamma_{k} \rightarrow 0^{+}$. That is, the generalized $K d V$ solitary waves are the limits in $H^{1}$ of solitary waves of the generalized Ostrovsky equation. 
To prove this theorem, we will show that the sequence of Ostrovsky solitary waves is a minimizing sequence for the $\mathrm{KdV}$ variational problem. The following lemma is proved in [15].

Lemma 2.6. The space $X_{1}$ is dense in $H^{1}$.

Using the lemma, we next prove that the function $m$ is continuous at $\gamma=0$.

Lemma 2.7. Fix $\beta>0$ and $c<0$. Then

$$
\lim _{\gamma \rightarrow 0^{+}} m(\beta, c, \gamma)=m(\beta, c, 0) .
$$

Proof. Since $m$ is strictly increasing in $\gamma$, it suffices to show that $m\left(\beta, c, \gamma_{k}\right) \rightarrow m(\beta, c, 0)$ for some sequence $\gamma_{k} \rightarrow 0$. By the density of $X_{1}$ in $H^{1}$ we may choose $\psi_{k}$ in $X_{1}$ such that $\left\|\psi_{k}-\varphi_{0}\right\|_{H^{1}}<\frac{1}{k}$ and define

$$
\gamma_{k}=\min \left(\frac{1}{k}, \frac{1}{k \int\left|D_{x}^{-1} \psi_{k}\right|^{2} d x}\right)
$$

Then

$$
\begin{aligned}
m\left(\beta, c, \gamma_{k}\right) & \leq \frac{I\left(\psi_{k} ; \beta, c, \gamma_{k}\right)}{K\left(\psi_{k}\right)^{\frac{2}{p+1}}} \\
& =\frac{I\left(\psi_{k} ; \beta, c, 0\right)+\gamma_{k} \int\left|D_{x}^{-1} \psi_{k}\right|^{2} d x}{K\left(\psi_{k}\right)^{\frac{2}{p+1}}} \\
& \leq \frac{I\left(\psi_{k} ; \beta, c, 0\right)+\frac{1}{k}}{K\left(\psi_{k}\right)^{\frac{2}{p+1}}} .
\end{aligned}
$$

Since $I(\cdot ; \beta, c, 0)$ and $K$ are both continuous on $H^{1}$, we therefore have

$$
\limsup _{k \rightarrow \infty} m\left(\beta, c, \gamma_{k}\right) \leq \frac{I\left(\varphi_{0} ; \beta, c, 0\right)}{K\left(\varphi_{0}\right)^{\frac{2}{p+1}}}=m(\beta, c, 0)
$$

On the other hand, given $\varphi_{k} \in G\left(\beta, c, \gamma_{k}\right)$ we have $\varphi_{k} \in H^{1}$, so

$$
\begin{aligned}
m(\beta, c, 0) & \leq \frac{I\left(\varphi_{k} ; \beta, c, 0\right)}{K\left(\varphi_{k}\right)^{\frac{2}{p+1}}} \\
& =\frac{I\left(\varphi_{k} ; \beta, c, \gamma_{k}\right)-\gamma_{k} \int\left|D_{x}^{-1} \varphi_{k}\right|^{2} d x}{K\left(\varphi_{k}\right)^{\frac{2}{p+1}}} \\
& <\frac{I\left(\varphi_{k} ; \beta, c, \gamma_{k}\right)}{K\left(\varphi_{k}\right)^{\frac{2}{p+1}}}=m\left(\beta, c, \gamma_{k}\right) .
\end{aligned}
$$

Thus

$$
\liminf _{k \rightarrow \infty} m\left(\beta, c, \gamma_{k}\right) \geq m(\beta, c, 0)
$$

and the lemma follows. 
Proof of Theorem 2.5. By continuity of $m$ at $\gamma=0$ we have

$$
\lim _{k \rightarrow \infty} K\left(\varphi_{k}\right)=\lim _{k \rightarrow \infty} m\left(\beta, c, \gamma_{k}\right)^{\frac{p+1}{p-1}}=m(\beta, c, 0)^{\frac{p+1}{p-1}}
$$

and

$$
\begin{aligned}
\limsup _{k \rightarrow \infty} I\left(\varphi_{k} ; \beta, c, 0\right) & =\limsup _{k \rightarrow \infty} I\left(\varphi_{k} ; \beta, c, \gamma_{k}\right)-\gamma_{k} \int\left(D_{x}^{-1} \varphi_{k}\right)^{2} d x \\
& \leq \lim _{k \rightarrow \infty} I\left(\varphi_{k} ; \beta, c, \gamma_{k}\right) \\
& =\lim _{k \rightarrow \infty} m\left(\beta, c, \gamma_{k}\right)^{\frac{p+1}{p-1}} \\
& =m(\beta, c, 0)^{\frac{p+1}{p-1}} .
\end{aligned}
$$

Thus $\varphi_{k}$ is a minimizing sequence for the $\mathrm{KdV}$ variational problem, and the result follows from Theorem 2.4.

\section{$3 \quad$ Stability}

The main result of this section is the following.

Theorem 3.1. Let $\beta>0, \gamma>0$ and $c<2 \sqrt{\beta \gamma}$. Let $d$ be defined as in (3.1). If $d^{\prime \prime}(c)>0$ then the set of ground states $G(\beta, c, \gamma)$ is $X_{1}$-stable.

The proof is based on arguments in [14], which makes use of the method of [5]. We remark here that the condition $d^{\prime \prime}(c)>0$ may be replaced by strict convexity of $d$ in a neighborhood of $c$. See [24].

Given $\beta>0, \gamma>0$ and $c<2 \sqrt{\beta \gamma}$, we define

$$
d(c)=d(\beta, c, \gamma)=E(\varphi)-c V(\varphi)
$$

where $\varphi$ is any element of $G(\beta, c, \gamma)$. Since

$$
E(u)-c V(u)=\frac{1}{2} I(u)-\frac{1}{p+1} K(u)
$$

it follows from (2.8) that

$$
d(\beta, c, \gamma)=\frac{p-1}{2(p+1)} I(\varphi)=\frac{p-1}{2(p+1)} K(\varphi)=\frac{p-1}{2(p+1)}(m(\beta, c, \gamma))^{\frac{p+1}{p-1}}
$$

Therefore $d$ is well-defined, and we may deduce its properties by examining the function $m(\beta, c, \gamma)$. The following two lemmas are immediate corollaries of Lemmas 2.2 and 2.3.

Lemma 3.2. Let $\beta>0, \gamma>0$ and $c<2 \sqrt{\beta \gamma}$. For any $r>0$ and $s>0$ we have

$$
d\left(r s^{2} \beta, r c, r s^{-2} \gamma\right)=r^{\frac{p+1}{p-1}} s d(\beta, c, \gamma)
$$


Lemma 3.3. The function $d$ is continuous on the domain $\beta>0, \gamma>0, c<2 \sqrt{\gamma \beta}$. Furthermore, $d$ is strictly increasing in $\gamma$ and $\beta$ and strictly decreasing in $c$.

Lemma 3.4. For each fixed $\beta>0$ and $\gamma>0$, the partial derivative $\partial d / \partial c(\beta, c, \gamma)$ exists for all but countably many $c<2 \sqrt{\beta \gamma}$. Similarly, $\partial d / \partial \beta$ and $\partial d / \partial \gamma$ exist for all but countably many $\beta$ and $\gamma$, respectively. At points where the partials exist,

$$
\begin{aligned}
& \frac{\partial d}{\partial \beta}=\frac{1}{2} \int\left(\varphi_{x}\right)^{2} d x \\
& \frac{\partial d}{\partial c}=-\frac{1}{2} \int \varphi^{2} d x \\
& \frac{\partial d}{\partial \gamma}=\frac{1}{2} \int\left(D_{x}^{-1} \varphi\right)^{2} d x .
\end{aligned}
$$

Proof. Since $d$ is continuous and monotone with respect to each variable, it follows that the partial derivatives exist at all but countable many points. To verify the formulas above, first fix $\beta>0$ and $\gamma>0$. Then by the inequalities in the proof of Lemma 2.3,

$$
-\frac{\int \varphi_{c_{2}}^{2} d x}{K\left(\varphi_{c_{2}}\right)^{\frac{2}{p+1}}} \leq \frac{m\left(\beta, c_{2}, \gamma\right)-m\left(\beta, c_{1}, \gamma\right)}{c_{2}-c_{1}} \leq-\frac{\int \varphi_{c_{1}}^{2} d x}{K\left(\varphi_{c_{1}}\right)^{\frac{2}{p+1}}}
$$

for $c_{1}<c_{2}<2 \sqrt{\beta \gamma}$. Let

$$
\begin{aligned}
& g_{s}(\beta, c, \gamma)=\sup \left\{\int \varphi_{c}^{2} d x \mid \varphi_{c} \in G(\beta, c, \gamma)\right\} \\
& g_{i}(\beta, c, \gamma)=\inf \left\{\int \varphi_{c}^{2} d x \mid \varphi_{c} \in G(\beta, c, \gamma)\right\}
\end{aligned}
$$

Then, for $c_{1}<c_{2}<2 \sqrt{\beta \gamma}$,

$$
-\frac{g_{i}\left(\beta, c_{2}, \gamma\right)}{m\left(\beta, c_{2}, \gamma\right)^{\frac{2}{p-1}}} \leq \frac{m\left(\beta, c_{2}, \gamma\right)-m\left(\beta, c_{1}, \gamma\right)}{c_{2}-c_{1}} \leq-\frac{g_{s}\left(\beta, c_{1}, \gamma\right)}{m\left(\beta, c_{1}, \gamma\right)^{\frac{2}{p-1}}}
$$

We now claim that

$$
\lim _{c \rightarrow c_{0}} \sup g_{i}(\beta, c, \gamma) \leq g_{s}\left(\beta, c_{0}, \gamma\right)
$$

To see this, choose any $c_{k} \rightarrow c_{0}$ and $\varphi_{k} \in G\left(\beta, c_{k}, \gamma\right)$. The continuity of $m$, the characterization (2.8) and the relation (3.3) imply that $I\left(\varphi_{k}\right) \rightarrow \frac{2(p+1)}{p-1} d\left(\beta, c_{0}, \gamma\right)$ and $K\left(\varphi_{k}\right) \rightarrow$ $\frac{2(p+1)}{p-1} d\left(\beta, c_{0}, \gamma\right)$. Therefore $\varphi_{k}$ is a minimizing sequence, so by Theorem 2.1 , there is a translated subsequence (renamed $\varphi_{k}$ ) which converges in $X_{1}$ to some function $\varphi$ in $G\left(\beta, c_{0}, \gamma\right)$. Hence

$$
\lim _{k \rightarrow \infty} \sup g_{i}\left(\beta, c_{k}, \gamma\right) \leq \int \varphi^{2} d x \leq g_{s}\left(\beta, c_{0}, \gamma\right)
$$

Consequently

$$
\frac{\partial m}{\partial c}\left(\beta, c^{+}, \gamma\right)=-\frac{g_{s}(\beta, c, \gamma)}{m(\beta, c, \gamma)^{\frac{2}{p-1}}}
$$


Now, since $d=\frac{p-1}{2(p+1)} m^{\frac{p+1}{p-1}}$, this implies

$$
\frac{\partial d}{\partial c}\left(\beta, c^{+}, \gamma\right)=-\frac{1}{2} g_{s}(\beta, c, \gamma)
$$

Likewise,

$$
\frac{\partial d}{\partial c}\left(\beta, c^{-}, \gamma\right)=-\frac{1}{2} g_{i}(\beta, c, \gamma)
$$

So at points where the partial derivative exists, we must have $g_{s}(\beta, c, \gamma)=g_{i}(\beta, c, \gamma)$, and the first formula above follows. The proof of the other formulas is similar.

We note here that the preceding proof illustrates that uniqueness of ground states up to translation would imply differentiability of $d$. For if $G(\beta, c, \gamma)$ consists of translates of a single ground state, then $g_{s}(\beta, c, \gamma)=g_{i}(\beta, c, \gamma)$, from which the differentiability of $d$ follows.

For the remainder of this section we will regard $d$ only as a function of $c$ for fixed $\beta$ and $\gamma$. So notation such as $d^{\prime}, d^{\prime \prime}$ or $d^{-1}$ should be interpreted with respect to the variable $c$. A key role in the stability analysis is played by the $\epsilon$-neighborhood of the set of ground states, defined by

$$
U_{c, \epsilon}=\left\{u \in X_{1} \mid \inf _{\varphi \in G(\beta, c, \gamma)}\|u-\varphi\|_{X_{1}}<\epsilon\right\} .
$$

Since $d$ is strictly decreasing in $c$, we may define

$$
c(u)=d^{-1}\left(\frac{p-1}{2(p+1)} K(u)\right) .
$$

This associates a speed $c(u)$ to any function $u \in X_{1}$. The following lemma provides the key estimate involving these speeds.

Lemma 3.5. If $d^{\prime \prime}(c)>0$ then there is some $\epsilon>0$ such that for any $u \in U_{c, \epsilon}$ and $\varphi \in$ $G(\beta, c, \gamma)$ we have

$$
E(u)-E(\varphi)-c(u)(V(u)-V(\varphi)) \geq \frac{1}{4} d^{\prime \prime}(c)|c(u)-c|^{2} .
$$

Proof. Since $d^{\prime}(c)=-V\left(\varphi_{c}\right)$, it follows from Taylor's theorem that

$$
d\left(c_{1}\right)=d(c)-V\left(\varphi_{c}\right)\left(c_{1}-c\right)+\frac{1}{2} d^{\prime \prime}(c)\left(c_{1}-c\right)^{2}+o\left(\left|c_{1}-c\right|^{2}\right)
$$

for $c_{1}$ near $c$. By choosing $\epsilon$ sufficiently small, it then follows that

$$
\begin{aligned}
d(c(u)) & \geq d(c)-V\left(\varphi_{c}\right)(c(u)-c)+\frac{1}{4} d^{\prime \prime}(c)(c(u)-c)^{2} \\
& =E\left(\varphi_{c}\right)-c(u) V\left(\varphi_{c}\right)+\frac{1}{4} d^{\prime \prime}(c)(c(u)-c)^{2}
\end{aligned}
$$

for $u \in U_{c, \epsilon}$. Now if $\varphi_{c(u)} \in G(\beta, c(u), \gamma)$ then

$$
K\left(\varphi_{c(u)}\right)=\frac{2(p+1)}{p-1} d(c(u))=K(u)
$$


and $\varphi_{c(u)}$ minimizes $I(\cdot ; \beta, c(u), \gamma)$ subject to this constraint, so

$$
\begin{aligned}
E(u)-c(u) V(u) & =\frac{1}{2} I(u ; \beta, c(u), \gamma)-\frac{1}{p+1} K(u) \\
& \geq \frac{1}{2} I\left(\varphi_{c(u)} ; \beta, c(u), \gamma\right)-\frac{1}{p+1} K\left(\varphi_{c(u)}\right) \\
& =d(c(u)) .
\end{aligned}
$$

This completes the proof.

Proof of Theorem 3.1. Suppose $G(\beta, c, \gamma)$ is $X_{1}$-unstable, and choose initial data $u_{0}^{k}$ such that

$$
\inf _{\varphi \in G(\beta, c, \gamma)}\left\|u_{0}^{k}-\varphi\right\|_{X_{1}}<\frac{1}{k}
$$

and let $u^{k}(t)$ be the solution of (1.2) with $u^{k}(0)=u_{0}^{k}$. By continuity in $t$, there is some $\delta>0$ and some times $t_{k}$ such that

$$
\inf _{\varphi \in G(\beta, c, \gamma)}\left\|u^{k}\left(t_{k}\right)-\varphi\right\|_{X_{1}}=\delta
$$

By the initial assumption, we can find $\varphi_{k} \in G(\beta, c, \gamma)$ such that

$$
\lim _{k \rightarrow \infty}\left\|u_{0}^{k}-\varphi_{k}\right\|_{X_{1}}=0 \text {. }
$$

Therefore, since $E$ and $V$ are continuous on $X_{1}$ and invariants of (1.2),

$$
\lim _{k \rightarrow \infty} E\left(u^{k}\left(t_{k}\right)\right)-E\left(\varphi_{k}\right)=\lim _{k \rightarrow \infty} E\left(u_{0}^{k}\right)-E\left(\varphi_{k}\right)=0
$$

and

$$
\lim _{k \rightarrow \infty} V\left(u^{k}\left(t_{k}\right)\right)-V\left(\varphi_{k}\right)=\lim _{k \rightarrow \infty} V\left(u_{0}^{k}\right)-V\left(\varphi_{k}\right)=0 .
$$

By Lemma 3.5, if $\delta$ is sufficiently small, we have

$$
E\left(u^{k}\left(t_{k}\right)\right)-E\left(\varphi_{k}\right)-c\left(u^{k}\left(t_{k}\right)\right)\left(V\left(u^{k}\left(t_{k}\right)\right)-V\left(\varphi_{k}\right)\right) \geq \frac{1}{4} d^{\prime \prime}(c)\left|c\left(u^{k}\left(t_{k}\right)\right)-c\right|^{2} .
$$

By equation (3.4) there is some $\psi_{k} \in G(\beta, c, \gamma)$ such that $\left\|u^{k}\left(t_{k}\right)-\psi_{k}\right\|_{X_{1}}<2 \delta$, and by (2.10), we have

$$
\left\|u^{k}\left(t_{k}\right)\right\|_{X_{1}} \leq\left\|\psi_{k}\right\|_{X_{1}}+2 \delta \leq 2 \delta+A^{-1} I\left(\psi_{k} ; \beta, c, \gamma\right)=2 \delta+\frac{2(p+1)}{A(p-1)} d(c)<\infty .
$$

Thus since $K$ is Lipschitz continuous on $X_{1}$ and $d^{-1}$ is continuous, it follows that $c\left(u^{k}\left(t_{k}\right)\right)$ is uniformly bounded in $k$. Thus by (3.5), (3.6) and (3.7) it follows that

$$
\lim _{k \rightarrow \infty} c\left(u^{k}\left(t_{k}\right)\right)=c .
$$

Continuity of $K$ then implies

$$
\lim _{k \rightarrow \infty} K\left(u^{k}\left(t_{k}\right)\right)=\lim _{k \rightarrow \infty} \frac{2(p+1)}{p-1} d\left(c\left(u^{k}\left(t_{k}\right)\right)\right)=\frac{2(p+1)}{p-1} d(c) .
$$


By (3.2) and (3.1), we have

$$
\begin{aligned}
\frac{1}{2} I\left(u^{k}\left(t_{k}\right)\right) & =E\left(u^{k}\left(t_{k}\right)\right)-c V\left(u^{k}\left(t_{k}\right)\right)+\frac{1}{p+1} K\left(u^{k}\left(t_{k}\right)\right) \\
& =d(c)+E\left(u^{k}\left(t_{k}\right)\right)-E\left(\varphi_{k}\right)-c\left(V\left(u^{k}\left(t_{k}\right)\right)-V\left(\varphi_{k}\right)\right)+\frac{1}{p+1} K\left(u^{k}\left(t_{k}\right)\right)
\end{aligned}
$$

so it follows from (3.5), (3.6) and (3.8) that

$$
\lim _{k \rightarrow \infty} I\left(u^{k}\left(t_{k}\right)\right)=\frac{2(p+1)}{p-1} d(c) .
$$

Thus $u^{k}\left(t_{k}\right)$ is a minimizing sequence and therefore has a subsequence which converges in $X_{1}$ to some $\varphi \in G(\beta, c, \gamma)$. This contradicts (3.4), so the proof of the theorem is complete.

\section{Instability}

In this section we present two theorems which provide conditions for orbital instability of solitary waves. The first is complementary to the stability theorem, in that it guarantees instability when $d^{\prime \prime}(c)<0$. The second does not involve the function $d$, but rather gives a set of sufficient conditions for instability directly in terms of the parameters $\beta, \gamma, c$ and $p$. While this result is not sharp, it does not rely on detailed knowledge of the function $d$. The proof is based on the work of Goncalves Rebeiro [10], which is a modification of Shatah and Strauss' method [25].

The first theorem requires the following assumption.

Assumption 4.1. For each fixed $\beta>0$ and $\gamma>0$, there exists a $C^{1}$ map $c \mapsto \varphi_{c}$ from $(-\infty, 2 \sqrt{\beta \gamma})$ to $X_{1}$ such that $\varphi_{c} \in G(\beta, c, \gamma)$.

Theorem 4.2. Suppose $\beta>0, \gamma>0, c<2 \sqrt{\beta \gamma}$ and Assumption 4.1 holds. If $d^{\prime \prime}(c)<0$, then the orbit $\mathcal{O}_{\varphi_{c}}$ is $X_{1}$-unstable.

Theorem 4.3. Let $\beta>0, \gamma>0, c<2 \sqrt{\beta \gamma}$ and $\varphi \in G(\beta, c, \gamma)$. Then the orbit $\mathcal{O}_{\varphi}$ is $X_{1}$-unstable if

(i) $c<0, p>5$, and $\gamma<\gamma_{0}$, for some small $\gamma_{0}>0$,

(ii) $c \leq 0$ and $p>5+4 \sqrt{2}$, and $\gamma>0$ or

(iii) $c>0$ and $p>\frac{10+k+\sqrt{(10+k)^{2}+4(7+k)}}{2}$, where $k=8\left(\frac{2 \sqrt{\beta \gamma}}{2 \sqrt{\beta \gamma}-c}-1\right)>0$.

Theorems 4.2 and 4.3 are actually both direct corollaries of the following Theorem 4.4, with different choices of the "unstable direction" $\phi$. The choices are given in Lemmas 4.10 
and 4.11. As in the proof of the stability theorem, an important role will be played by the $\epsilon$-neighborhoods of the orbits of solitary waves. Given $\varphi \in G(\beta, c, \gamma)$ and $\epsilon>0$, we define

$$
U_{\varphi, \epsilon}=\left\{u \in X_{1} \mid \inf _{v \in \mathcal{O}_{\varphi}}\|u-v\|_{X_{1}}<\epsilon\right\}
$$

Theorem 4.4. Assume $\beta>0, \gamma>0$ and $c<2 \sqrt{\beta \gamma}$. Let $\mathcal{M}=\left\{u \in X_{1} ; V(u)=V\left(\varphi_{c}\right)\right\}$ where $\varphi=\varphi_{c} \in G(\beta, c, \gamma)$. If there exists $\phi \in L^{2}$, such that $\phi^{\prime} \in X_{s}, s>3 / 2, \phi^{\prime \prime} \in X_{1}$, $\phi^{\prime}$ is tangent to $\mathcal{M}$ at $\varphi$, and

$$
\left\langle L^{\prime \prime}(\varphi) \phi^{\prime}, \phi^{\prime}\right\rangle<0,
$$

then there exists an $\epsilon>0$ and a sequence $\left\{u_{0}^{j}\right\}$ in $U_{\varphi, \epsilon}$ such that

(i) $u_{0}^{j} \rightarrow \varphi$ in $X_{1}$ as $j \rightarrow \infty$.

(ii) For all positive integer $j, u^{j}$ is uniformly bounded, but escapes $U_{\varphi, \epsilon}$ in finite time, where $u^{j}$ is the solution of $(1.2)$ with $u^{j}(0)=u_{0}^{j}$.

The proof of Theorem 4.4 is approached via a series of lemmas. Define

$$
J(u)=\int \beta u_{x}^{2}+\gamma\left(D_{x}^{-1} u\right)^{2}-c u^{2}+(p+1) F(u) d x
$$

and

$$
L(u)=E(u)-c V(u)=\frac{1}{2} J(u)+\frac{p-1}{2(p+1)} K(u) .
$$

Lemma 4.5. Assume $c<2 \sqrt{\beta \gamma}$. Then there exists a ground state $\varphi \in X_{1}$ satisfying $J(\varphi)=0$ such that

$$
L(\varphi)=\inf \left\{L(u) \mid u \in X_{1}, u \neq 0, J(u)=0\right\} .
$$

Proof. The Lemma follows by applying the arguments in the proof of Proposition 2.3 in $[17]$.

Lemma 4.6. Fix $c<2 \sqrt{\gamma \beta}$, let $\varphi \in G(\beta, c, \gamma)$. There is an $\epsilon_{0}>0$ and a unique $C^{2}$ map $\alpha: U_{\varphi, \epsilon_{0}} \rightarrow \mathbf{R}$ such that $\alpha(\varphi)=0$ and for all $v \in U_{\varphi, \epsilon_{0}}$, and any $r \in \mathbf{R}$,

(i) $\left\langle\tau_{\alpha(v)} \varphi^{\prime}, v\right\rangle=0$

(ii) $\alpha\left(\tau_{r} v\right)=\alpha(v)+r$, and

(iii) $\alpha^{\prime}(v)=-\frac{1}{\left\langle v, \varphi^{\prime \prime}(\cdot+\alpha(v))\right\rangle} \varphi^{\prime}(\cdot+\alpha(v))$.

In particular, for any $w \in \mathcal{O}_{\varphi}$ we have $\left\langle\alpha^{\prime}(w), w\right\rangle=0$, and $\alpha^{\prime}(w)=\frac{1}{\left|\varphi^{\prime}\right|_{2}^{2}} w^{\prime}$.

Proof. The proof is standard. See Theorem 3.1 in [10], Lemma 3.5 in [1], or Lemma 3.5 in [3]. 
Consider a function $\phi \in L^{2}$ such that $\phi^{\prime} \in X_{1}$. Define another vector field $B_{\phi}$ by

$$
B_{\phi}(v)=\tau_{\alpha(v)} \phi^{\prime}-\frac{\left\langle v, \tau_{\alpha(v)} \phi^{\prime}\right\rangle}{\left\langle v, \tau_{\alpha(v)} \varphi^{\prime \prime}\right\rangle} \tau_{\alpha(v)} \varphi^{\prime \prime}
$$

for $v \in U_{\varphi, \epsilon}$.

The vector field $B_{\phi}$ is an extension of the formula (4.2) in Bona, Souganidis and Strauss [5] and a similar formula was also used in $[1,3,10]$. The important properties of $B_{\phi}$ are expressed in the following auxiliary result and will be used in the proof of Theorem 4.4.

Lemma 4.7. (i) Assume that $\phi \in L^{2}$ such that $\phi^{\prime}, \phi^{\prime \prime} \in X_{1}$. The mapping $B_{\phi}: U_{\varphi, \epsilon_{0}} \rightarrow X_{1}$ is $C^{1}$ with bounded derivative.

(ii) $B_{\phi}$ commutes with translations.

(iii) $\left\langle B_{\phi}(v), v\right\rangle=0$, for all $v \in U_{\varphi, \epsilon_{0}}$.

(iv) If $\left\langle\varphi, \phi^{\prime}\right\rangle=0$, then $B_{\phi}(\varphi)=\phi^{\prime}$.

Proof. Since $\phi^{\prime}, \varphi^{\prime \prime} \in X_{1}$, it is easy to see from the definition of $B_{\phi}$ that $B_{\phi}(v) \in X_{1}$ for all $v \in U_{\varphi, \epsilon}$. We now prove that $B_{\phi}$ is $C^{1}$ with bounded derivative. From part (iii) of Lemma 4.6 and (4.4), we have

$$
B_{\phi}(v)=\tau_{\alpha(v)} \phi^{\prime}+\left\langle v, \tau_{\alpha(v)} \phi^{\prime}\right\rangle \frac{d}{d x} \alpha^{\prime}(v) .
$$

A simple calculation shows

$$
\begin{aligned}
B_{\phi}^{\prime}(v) w & =\left\langle\alpha^{\prime}(v), w\right\rangle \tau_{\alpha(v)} \phi^{\prime \prime}+\left(\left\langle w, \tau_{\alpha(v)} \phi^{\prime}\right\rangle+\left\langle\alpha^{\prime}(v), w\right\rangle\left\langle v, \tau_{\alpha(v)} \phi^{\prime \prime}\right\rangle\right) \frac{d}{d x} \alpha^{\prime}(v) \\
& +\left\langle v, \tau_{\alpha(v)} \phi^{\prime}\right\rangle \frac{d}{d x} \alpha^{\prime \prime}(v) w
\end{aligned}
$$

for all $w \in X_{1}$. To show $B_{\phi}$ is a $C^{1}$ function with bounded derivative, we need to show that all terms in the right side of (4.5) are bounded in $U_{\varphi, \epsilon}$. In fact, for $w \in X_{1}$ and $v \in U_{\varphi, \epsilon}$, we have

$$
\begin{aligned}
\left\langle v^{\prime}, \tau_{\alpha(v)} \varphi^{\prime}\right\rangle \frac{d}{d x} \alpha^{\prime \prime}(v) w & =\left\langle\alpha^{\prime}(v), w\right\rangle \tau_{\alpha(v)} \varphi^{\prime \prime \prime} \\
& +\left\langle\tau_{\alpha(v)} \varphi^{\prime \prime}, w\right\rangle \frac{d}{d x} \alpha^{\prime}(v)-\left\langle\alpha^{\prime}(v), w\right\rangle\left\langle v^{\prime}, \tau_{\alpha(v)} \varphi^{\prime \prime}\right\rangle \frac{d}{d x} \alpha^{\prime}(v)
\end{aligned}
$$

Setting $v=\varphi$ in (4.6) and using the relation $\alpha(\varphi)=0$ yields

$$
\frac{d}{d x} \alpha^{\prime \prime}(\varphi) w=\frac{\varphi^{\prime \prime \prime}}{\left|\varphi^{\prime}\right|_{2}^{4}}\left\langle w, \varphi^{\prime}\right\rangle+\frac{\varphi^{\prime \prime}}{\left|\varphi^{\prime}\right|_{2}^{4}}\left\langle w, \varphi^{\prime \prime}\right\rangle
$$

Therefore,

$$
\left\|\frac{d}{d x} \alpha^{\prime \prime}(\varphi)\right\|_{L\left(X_{1}, X_{1}\right)} \leq C_{0}\left(\|\varphi\|_{4}\right)
$$


and

$$
\left\|\frac{d}{d x} \alpha^{\prime}(\varphi)\right\|_{X_{1}} \leq C_{1}\left(\|\varphi\|_{3}\right)
$$

Since $\alpha$ is $C^{2}$ and $\frac{d}{d x} \alpha^{\prime}$ and $\frac{d}{d x} \alpha^{\prime \prime}$ are continuous, by taking $\epsilon>0$ small enough, if necessary, there exists a constant $C_{2}>0$ such that $\left\|\alpha^{\prime}(v)\right\|_{X_{1}} \leq C_{2},\left\|\frac{d}{d x} \alpha^{\prime}(v)\right\|_{X_{1}} \leq C_{2}$, and $\left\|\frac{d}{d x} \alpha^{\prime \prime}(v)\right\|_{L\left(X_{1}, X_{1}\right)} \leq C_{2}$ with $C_{2}=C_{2}\left(\|\varphi\|_{4}\right)$ and for all $v \in U_{\varphi, \epsilon_{0}}$. It follows that $B_{\phi}$ is a $C^{1}$ function and the derivative of $B_{\phi}$ is bounded by

$$
\left\|B_{\phi}^{\prime}(v) w\right\|_{X_{1}} \leq C_{2}\left(\left\|\phi^{\prime \prime}\right\|_{X_{1}}+\left(\epsilon+\|\varphi\|_{X_{1}}\right)\left|\phi^{\prime}\right|_{2}\right)\|w\|_{X_{1}}
$$

which implies that

$$
\left\|B_{\phi}^{\prime}(v)\right\|_{L\left(X_{1}, X_{1}\right)} \leq C, \quad \forall v \in U_{\varphi, \epsilon}
$$

where the constant $C$ depends only on $C_{2},\left\|\phi^{\prime \prime}\right\|_{X_{1}},\left|\phi^{\prime}\right|_{2}$, and $\|\varphi\|_{X_{1}}$. This proves (i). The statement (ii) can be obtained immediately from the relation $\alpha\left(\tau_{y}(v)\right)=\alpha(v)+y$ for any $v \in X_{1}$ and $y \in \mathbf{R}$. The statement (iii) can obtained directly from the definition of $B_{\phi}$. By $\alpha(\varphi)=0$ and by the assumption in (iv), $\left\langle\varphi, \phi^{\prime}\right\rangle=0$, we have

$$
B_{\phi}(\varphi)=\phi^{\prime}+\frac{\left\langle\varphi, \phi^{\prime}\right\rangle}{\left|\varphi^{\prime}\right|_{2}^{2}} \varphi^{\prime \prime}=\phi^{\prime}
$$

This completes the proof of Lemma 4.7.

Lemma 4.8. Let $\varphi \in G(\beta, c, \gamma)$. Assume that $\phi \in L^{2}$ is defined in Theorem 4.4. Then there exist $\epsilon_{3}>0$ and $\sigma_{3}>0$, such that for each $v_{0} \in U_{\varphi, \epsilon_{3}}$,

$$
L(\varphi) \leq L\left(v_{0}\right)+P\left(v_{0}\right) s
$$

for some $s \in\left(-\sigma_{3}, \sigma_{3}\right)$, where $P(v)=\left\langle L^{\prime}(v), B_{\phi}(v)\right\rangle$ and $L(v)=E(v)-c V(v)$.

Proof. Let $U_{\varphi, \epsilon}$ be as in Lemma 4.6. For each $v_{0} \in U_{\varphi, \epsilon_{0}}$, consider the initial-value problem

$$
\begin{aligned}
\frac{d v}{d s} & =B_{\phi}(v), \\
v(0) & =v_{0} .
\end{aligned}
$$

By Lemma 4.7, it has a unique maximal solution $v\left(v_{0}, s\right) \in C^{2}\left(U_{\varphi, \epsilon_{0}},(-\sigma, \sigma)\right)$ with $\sigma=$ $\sigma\left(v_{0}\right)>0$. Moreover, for each $\epsilon_{1}<\epsilon_{0}$, there exists $\sigma_{1}>0$, such that $\sigma\left(v_{0}\right) \geq \sigma_{1}$ for all $v_{0} \in$ $U_{\varphi, \epsilon_{1}}$. Hence, for fixed $\epsilon_{1}$ and $\sigma_{1}$, we can define the $C^{2}$-mapping $s \in\left(-\sigma_{1}, \sigma_{1}\right) \mapsto L\left(v\left(v_{0}, s\right)\right)$. Let $P(v)=\left\langle L^{\prime}(v), B_{\phi}(v)\right\rangle$ and

$$
R(v)=\left\langle L^{\prime \prime}(v) B_{\phi}(v), B_{\phi}(v)\right\rangle+\left\langle L^{\prime}(v), B_{\phi}^{\prime}(v)\left(B_{\phi}(v)\right)\right\rangle .
$$

Applying Taylor's theorem yields

$$
L\left(v\left(v_{0}, s\right)\right)=L\left(v_{0}\right)+P\left(v_{0}\right) s+\frac{1}{2} R\left(v\left(v_{0}, \xi s\right)\right) s^{2}
$$


for some $\xi \in(0,1)$. Since $P$ and $R$ are continuous, $L^{\prime}(\varphi)=0$, and $R(\varphi)=\left\langle L^{\prime \prime}(\varphi) \phi^{\prime}, \phi^{\prime}\right\rangle<0$, there exist $\epsilon_{2} \in\left(0, \epsilon_{1}\right)$ and $\sigma_{2} \in\left(0, \sigma_{1}\right)$ such that

$$
L\left(v\left(v_{0}, s\right)\right) \leq L\left(v_{0}\right)+P\left(v_{0}\right) s
$$

for $v_{0} \in B\left(\varphi, \epsilon_{2}\right)$ and $s \in\left(-\sigma_{2}, \sigma_{2}\right)$. On the other hand, a simple computation shows that

$$
\left.J\left(v\left(v_{0}, s\right)\right)\right|_{\left(v_{0}, s\right)=(\varphi, 0)}=0
$$

and

$$
\left.\frac{\partial}{\partial s} J\left(v\left(v_{0}, s\right)\right)\right|_{\left(v_{0}, s\right)=(\varphi, 0)}=\left\langle J^{\prime}(\varphi), \phi^{\prime}\right\rangle .
$$

We claim that $\left\langle J^{\prime}(\varphi), \phi^{\prime}\right\rangle \neq 0$. Otherwise, $\phi^{\prime}$ would be tangent to $\mathcal{N}$ at $\varphi$, where

$$
\mathcal{N}=\left\{u \in X_{1} \mid u \neq 0, J(u)=0\right\} .
$$

Hence, $\left\langle L^{\prime \prime}(\varphi) \phi^{\prime}, \phi^{\prime}\right\rangle \geq 0$ since $\varphi$ minimizes $L$ on $\mathcal{N}$ by Lemma 4.5. But this contradicts (4.1). Therefore, by the implicit function theorem, there exists $\epsilon_{3} \in\left(0, \epsilon_{2}\right)$ and $\sigma_{3} \in\left(0, \sigma_{2}\right)$ such that for every $v_{0} \in B\left(\varphi, \epsilon_{3}\right)$, there exists a unique $s=s\left(v_{0}\right) \in\left(-\sigma_{3}, \sigma_{3}\right)$ such that

$$
J\left(v\left(v_{0}, s\left(v_{0}\right)\right)\right)=0 .
$$

Applying (4.12) to $\left(v_{0}, s\left(v_{0}\right)\right)$ given by (4.14) and taking into account that $\varphi$ minimizes $L$ on $\mathcal{N}$, we have

$$
L(\varphi) \leq L\left(v\left(v_{0}, s\right)\right) \leq L\left(v_{0}\right)+P\left(v_{0}\right) s
$$

for some $s \in\left(-\sigma_{3}, \sigma_{3}\right)$. The above inequality can be extended to $U_{\varphi, \epsilon_{3}}$ from the gauge invariance.

Remark 4.9. From the relation

$$
v(\varphi, s)=\varphi+\int_{0}^{s} \tau_{\alpha(v(\varphi, t))} \phi^{\prime} d t-\int_{0}^{s} g(t) \tau_{\alpha(v(\varphi, t))} \varphi^{\prime \prime} d t
$$

where

$$
g(t)=\frac{\left\langle v, \tau_{\alpha(v)} \phi^{\prime}\right\rangle}{\left\langle v, \tau_{\alpha(v)} \varphi^{\prime \prime}\right\rangle},
$$

it is easy to see that $v(\varphi, s) \in X_{l}, l>\frac{3}{2}$, for all $s \in\left(-\sigma_{3}, \sigma_{3}\right)$.

Now we are in the position to prove Theorem 4.4 .

Proof of Theorem 4.4. Since $v\left(v_{0}, s\right)$ commutes with $\tau_{y}$, it follows by replacing $v_{0}$ with $v(\varphi, \delta)$ in (4.15) that

$$
L(v(v(\varphi, \delta), s)) \leq L(v(\varphi, \delta))+P(v(\varphi, \delta)) s
$$

for any $s \in\left(-\sigma_{2}, \sigma_{2}\right)$ and $\delta \in\left(-\sigma_{3}, \sigma_{3}\right)$ with $0<\sigma_{3}<\sigma_{2}$. Taking $s=-\delta$, it thus transpires from (4.16) that

$$
L(\varphi) \leq L(v(\varphi, \delta))-P(v(\varphi, \delta)) \delta
$$


for all $\delta \in\left(-\sigma_{3}, \sigma_{3}\right)$. Moreover, it follows from (4.11) and the fact that $P(\varphi)=0$ that the mapping $\delta \mapsto L(v(\varphi, \delta))$ has a strict maximum locally at $\delta=0$. Hence, we have

$$
L(v(\varphi, \delta))<L(\varphi)
$$

for all $\delta \neq 0$ and $\delta \in\left(-\sigma_{4}, \sigma_{4}\right)$ with $0<\sigma_{4} \leq \sigma_{3}$. This in turn implies from (4.17) that

$$
P(v(\varphi, \delta))<0
$$

for all $\delta \in\left(0, \sigma_{4}\right)$. Let $\delta_{j} \in\left(0, \sigma_{4}\right)$ such that $\delta_{j} \rightarrow 0$ as $j \rightarrow \infty$. Consider the sequences of initial data $u_{0, j}=v\left(\varphi, \delta_{j}\right)$. Then by Remark 4.9, $u_{0, j} \in X_{s}, s>3 / 2$, for all positive integers $j$ and $u_{0, j} \rightarrow \varphi$ in $X_{1}$ as $j \rightarrow \infty$, which proves (i). For all integers $j$, we need only verify the solution $u_{j}(t)$ of (1.2) with $u_{j}(0)=u_{0, j}$ escapes from $U_{\varphi, \epsilon_{3}}$ for some $\epsilon_{3}>0$ and for all positive integers $j$ in finite time. To see this, let $\epsilon_{3}$ be defined as in Lemma 4.8. Define

$$
T_{j}=\sup \left\{\lambda>0 ; \quad u_{j}(t) \in U_{\varphi, \epsilon_{3}}, \forall t \in(0, \lambda)\right\}
$$

and

$$
P_{-}=\left\{v \in U_{\varphi, \epsilon_{3}} ; \quad L(v)<L(\varphi), P(v)<0\right\} .
$$

Consider the case of the maximal existence time $T=+\infty$ by the definition of stability. It now follows from Lemma 4.8 that for all integers $j$ and $t \in\left(0, T_{j}\right)$, there exists $s=s_{j}(t) \in$ $\left(-\sigma_{3}, \sigma_{3}\right)$ satisfying

$$
L(\varphi) \leq L\left(u_{j}(t)\right)+P\left(u_{j}(t)\right) s=L\left(u_{0, j}\right)+P\left(u_{j}(t)\right) s .
$$

By (4.18) and (4.19), $u_{0, j} \in P_{-}$. Then we deduce that $u_{j}(t) \in P_{-}$for all $t \in\left[0, T_{j}\right]$. In fact, if $P\left(u_{j}\left(t_{0}\right)\right)>0$ for some $t_{0} \in\left[0, T_{j}\right]$, then the continuity of $P$ implies that there exists some $t_{1} \in\left[0, T_{j}\right]$ satisfying $P\left(u_{j}\left(t_{1}\right)\right)=0$ and it thus follows from $(4.20)$ that $L(\varphi) \leq L\left(u_{0, j}\right)$ which contradicts $u_{0, j} \in P_{-}$. Hence, by (4.20), $P$ is bounded away from zero and

$$
-P\left(u_{j}(t)\right) \geq \frac{L(\varphi)-L\left(u_{0, j}\right)}{\sigma_{3}}=\eta_{j}>0, \quad \forall t \in\left[0, T_{j}\right]
$$

we now define a Liapunov function

$$
A(t)=\int_{\mathbf{R}} \phi\left(x+\alpha\left(u_{j}(t)\right)\right) u_{j}(x, t) d x, \quad t \in\left[0, T_{j}\right] .
$$

Then by the Cauchy-Schwarz inequality,

$$
|A(t)| \leq|\phi|_{2}\left|u_{j}(t)\right|_{2}=|\phi|_{2}\left|u_{0, j}\right|_{2}<+\infty, \quad t \in\left[0, T_{j}\right] .
$$

On the other hand, using the Hamiltonian formulation

$$
\frac{d u_{j}}{d t}=-\partial_{x} E^{\prime}\left(u_{j}\right)
$$


of (1.2), we have

$$
\begin{aligned}
\frac{d A}{d t} & =\alpha^{\prime}\left(u_{j}(t)\right) \int_{\mathbf{R}} \phi^{\prime}\left(x+\alpha\left(u_{j}(t)\right)\right) u_{j}(t)+\int_{\mathbf{R}} \phi\left(x+\alpha\left(u_{j}(t)\right)\right) \frac{d u_{j}}{d t} \\
& =\left\langle\alpha^{\prime}\left(u_{j}(t)\right), \frac{d u_{j}}{d t}\right\rangle\left\langle\tau_{\alpha\left(u_{j}(t)\right)} \phi^{\prime}, u_{j}\right\rangle+\left\langle\tau_{\alpha\left(u_{j}(t)\right)} \phi, \frac{d u_{j}}{d t}\right\rangle \\
& =\left\langle\left\langle\tau_{\alpha\left(u_{j}(t)\right)} \phi^{\prime}, u_{j}(t)\right\rangle \frac{d \alpha^{\prime}\left(u_{j}(t)\right)}{d x}+\tau_{\alpha\left(u_{j}(t)\right)} \phi^{\prime}, E^{\prime}\left(u_{j}(t)\right)\right\rangle \\
& =\left\langle B_{\phi}\left(u_{j}(t)\right), E^{\prime}\left(u_{j}(t)\right)\right\rangle \\
& =\left\langle B_{\phi}\left(u_{j}(t)\right), L^{\prime}\left(u_{j}(t)\right)\right\rangle+c\left\langle B_{\phi}\left(u_{j}(t)\right), u_{j}(t)\right\rangle \\
& =P\left(u_{j}(t)\right)
\end{aligned}
$$

for $t \in\left[0, T_{j}\right]$, where $\left\langle B_{\phi}\left(u_{j}(t)\right), u_{j}(t)\right\rangle=0$. Hence (4.21) yields the lower bound

$$
-\frac{d A}{d t} \geq \eta_{j}>0 \quad \forall t \in\left[0, T_{j}\right]
$$

Comparing (4.23) and (4.25), we conclude that $T_{j}<+\infty$, for all $j$. This completes the proof.

In view of Theorem 4.4 we now look for functions $\phi$ that satisfies the inequality (4.1).

Lemma 4.10. Suppose $\beta>0, \gamma>0, c<2 \sqrt{\beta \gamma}$ and Assumption 4.1 holds. If $d^{\prime \prime}(c)<0$ then there exists $\phi$ satisfying the conditions of Theorem 4.4.

Proof. By Assumption 4.1, $d$ is differentiable and by Lemma 3.4, $d^{\prime}(c)=-V\left(\varphi_{c}\right)$. So if we define

$$
g(h, \sigma)=V\left(\varphi_{h}+\sigma \varphi_{c}\right)
$$

then $g(c, 0)=-d^{\prime}(c)$ and

$$
\frac{\partial g}{\partial h}(c, 0)=\left\langle V^{\prime}\left(\varphi_{c}\right), \frac{\partial \varphi_{c}}{\partial c}\right\rangle=-d^{\prime \prime}(c)>0
$$

Therefore the implicit function theorem implies that there is a $C^{2}$ map $h:(-\epsilon, \epsilon) \rightarrow$ $(-\infty, 2 \sqrt{\beta \gamma})$ such that $h(0)=c$ and

$$
g(h(\sigma), \sigma)=V\left(\varphi_{h(\sigma)}+\sigma \varphi_{c}\right)=V\left(\varphi_{c}\right) .
$$

Thus

$$
0=\left.\frac{d}{d \sigma} g(h(\sigma), \sigma)\right|_{\sigma=0}=\left\langle V^{\prime}\left(\varphi_{c}\right), h^{\prime}(0) \frac{\partial \varphi_{c}}{\partial c}+\varphi_{c}\right\rangle
$$

so if we define

$$
\phi(x)=\int_{-\infty}^{x} h^{\prime}(0) \frac{\partial \varphi_{c}}{\partial c}(y)+\varphi_{c}(y) d y
$$

then $\phi^{\prime}$ is tangent to $\mathcal{M}$ at $\varphi_{c}$ and it follows from Assumption 4.1 that $\phi \in L^{2}, \phi^{\prime} \in X_{s}$ for some $s>3 / 2$ and $\phi^{\prime \prime} \in X_{1}$. It remains to show that $\phi$ satisfies (4.1). First observe that

$$
L^{\prime \prime}(\varphi)=E^{\prime \prime}\left(\varphi_{c}\right)-c V^{\prime \prime}\left(\varphi_{c}\right)=-\beta \partial_{x}^{2}-\gamma D_{x}^{-2}-c+f^{\prime}\left(\varphi_{c}\right)
$$


SO

$$
\left\langle L^{\prime \prime}\left(\varphi_{c}\right) \phi^{\prime}, \phi^{\prime}\right\rangle=\left\langle L^{\prime \prime}\left(\varphi_{c}\right) \varphi_{c}, \varphi_{c}\right\rangle+2 h^{\prime}(0)\left\langle L^{\prime \prime}\left(\varphi_{c}\right) \varphi_{c}, \frac{\partial \varphi_{c}}{\partial c}\right\rangle+\left(h^{\prime}(0)\right)^{2}\left\langle L^{\prime \prime}\left(\varphi_{c}\right) \frac{\partial \varphi_{c}}{\partial c}, \frac{\partial \varphi_{c}}{\partial c}\right\rangle .
$$

We claim that

$$
\begin{gathered}
\left\langle L^{\prime \prime}\left(\varphi_{c}\right) \varphi_{c}, \varphi_{c}\right\rangle=(1-p) K\left(\varphi_{c}\right), \\
\left\langle L^{\prime \prime}\left(\varphi_{c}\right) \varphi_{c}, \frac{\partial \varphi_{c}}{\partial c}\right\rangle=-2 d^{\prime}(c),
\end{gathered}
$$

and

$$
\left\langle L^{\prime \prime}\left(\varphi_{c}\right) \frac{\partial \varphi_{c}}{\partial c}, \frac{\partial \varphi_{c}}{\partial c}\right\rangle=-d^{\prime \prime}(c)
$$

To prove the the first two identities, recall that $s f^{\prime}(s)=p f(s)$ and $F^{\prime}=f$. Thus

$$
\begin{aligned}
\left\langle L^{\prime \prime}\left(\varphi_{c}\right) \varphi_{c}, \varphi_{c}\right\rangle & =\int_{\mathbf{R}}\left(-\beta\left(\varphi_{c}\right)_{x x}-\gamma D_{x}^{-2} \varphi_{c}-c \varphi_{c}+p f\left(\varphi_{c}\right)\right) \varphi_{c} d x \\
& =\int_{\mathbf{R}}(p-1) \varphi_{c} f\left(\varphi_{c}\right) d x \\
& =(1-p) K\left(\varphi_{c}\right),
\end{aligned}
$$

and

$$
\begin{aligned}
\left\langle L^{\prime \prime}\left(\varphi_{c}\right) \varphi_{c}, \frac{\partial \varphi_{c}}{\partial c}\right\rangle & =\int_{\mathbf{R}}\left(-\beta\left(\varphi_{c}\right)_{x x}-\gamma D_{x}^{-2} \varphi_{c}-c \varphi_{c}+p f\left(\varphi_{c}\right)\right) \frac{\partial \varphi_{c}}{\partial c} d x \\
& =\int_{\mathbf{R}}(p-1) f\left(\varphi_{c}\right) \frac{\partial \varphi_{c}}{\partial c} d x \\
& =(p-1) \frac{d}{d c} \int_{\mathbf{R}} F\left(\varphi_{c}\right) d x \\
& =-2 d^{\prime}(c) .
\end{aligned}
$$

For the third identity, differentiate the solitary wave equation with respect to $c$ to find

$$
\beta\left(\frac{\partial \varphi_{c}}{\partial c}\right)_{x x}+c \frac{\partial \varphi_{c}}{\partial c}+\varphi_{c}+\gamma D_{x}^{-2} \frac{\partial \varphi_{c}}{\partial c}=f^{\prime}(\varphi) \frac{\partial \varphi_{c}}{\partial c},
$$

so $L^{\prime \prime}\left(\varphi_{c}\right)\left(\frac{\partial \varphi_{c}}{\partial c}\right)=\varphi_{c}$, and therefore

$$
\left\langle L^{\prime \prime}\left(\varphi_{c}\right) \frac{\partial \varphi_{c}}{\partial c}, \frac{\partial \varphi_{c}}{\partial c}\right\rangle=\left\langle\varphi_{c}, \frac{\partial \varphi_{c}}{\partial c}\right\rangle=\frac{1}{2} \frac{d}{d c} \int_{\mathbf{R}} \varphi_{c}^{2} d x=-d^{\prime \prime}(c)
$$

by Lemma 3.4. We next compute $h^{\prime}(0)$. Using (4.26) and Lemma 3.4, we have

$$
0=h^{\prime}(0)\left\langle\varphi_{c}, \frac{\partial \varphi_{c}}{\partial c}\right\rangle+\left\langle\varphi_{c}, \varphi_{c}\right\rangle=\frac{1}{2} h^{\prime}(0) \frac{d}{d c}\left\langle\varphi_{c}, \varphi_{c}\right\rangle+\left\langle\varphi_{c}, \varphi_{c}\right\rangle=-\frac{1}{2} h^{\prime}(0) d^{\prime \prime}(c)-d^{\prime}(c)
$$

and therefore

$$
h^{\prime}(0)=-\frac{2 d^{\prime}(c)}{d^{\prime \prime}(c)}
$$


Finally, equations (4.28), (4.29) and (4.30) give

$$
\left\langle L^{\prime \prime}\left(\varphi_{c}\right) \phi^{\prime}, \phi^{\prime}\right\rangle=(1-p) K\left(\varphi_{c}\right)+4 \frac{\left(d^{\prime}(c)\right)^{2}}{d^{\prime \prime}(c)}<0
$$

under the assumption that $d^{\prime \prime}(c)<0$. This proves the lemma.

Lemma 4.11. Assume $c<2 \sqrt{\beta \gamma}$ and let $\varphi \in G(\beta, c, \gamma)$. Define

$$
\phi(x)=\int_{-\infty}^{x}\left(\varphi(y)+2 y \varphi^{\prime}(y)\right) d y .
$$

Then

(i) $\phi \in L^{2}, \phi^{\prime} \in X_{s}, s>3 / 2, \phi^{\prime \prime} \in X_{1}$, and $\phi^{\prime}$ is tangent to $\mathcal{M}$ at $\varphi$.

(ii) $\left\langle L^{\prime \prime}(\varphi) \phi^{\prime}, \phi^{\prime}\right\rangle=\frac{(p-1)(5-p)}{p+1} K(\varphi)+16 \gamma \int_{\mathbf{R}}\left(D_{x}^{-1} \varphi\right)^{2} d x$.

Proof. The first part of statement (i) is obvious because $\varphi \in X_{s}$ for $s>3 / 2$ and $\varphi$ is exponentially decaying at infinity. On the other hand, a simple calculation shows that

$$
\left\langle\phi^{\prime}, \varphi\right\rangle=\int_{\mathbf{R}}\left(\varphi(x)+2 x \varphi^{\prime}(x)\right) \varphi(x) d x=\int_{\mathbf{R}}\left(x \varphi^{2}\right)^{\prime} d x=0 .
$$

This proves (i). Now we need to estimate the quantity $\left\langle L^{\prime \prime}(\varphi) \phi^{\prime}, \phi^{\prime}\right\rangle$. Differentiating the solitary wave equation

$$
\beta \varphi_{x x}+c \varphi+\gamma D_{x}^{-2} \varphi=f(\varphi)
$$

gives

$$
\beta \varphi_{x x x}+c \varphi_{x}+\gamma D_{x}^{-1} \varphi=f^{\prime}(\varphi) \varphi_{x}
$$

We now claim that

$$
\left\langle L^{\prime \prime}(\varphi) \varphi, x \varphi^{\prime}\right\rangle=\frac{p-1}{p+1} K(\varphi)
$$

and

$$
\left\langle L^{\prime \prime}(\varphi)\left(x \varphi^{\prime}\right), x \varphi^{\prime}\right\rangle=\frac{p-1}{2(p+1)} K(\varphi)+4 \gamma \int_{\mathbf{R}}\left(D_{x}^{-1} \varphi\right)^{2} .
$$

To prove these, again recall that $f^{\prime}(\varphi) \varphi=p f(\varphi)$ and $F^{\prime}=f$, so that

$$
\begin{aligned}
\left\langle L^{\prime \prime}(\varphi) \varphi, x \varphi^{\prime}\right\rangle & =\int_{\mathbf{R}}\left(-\beta \varphi_{x x}-\gamma D_{x}^{-2} \varphi-c \varphi+p f(\varphi)\right) x \varphi^{\prime} d x \\
& =\int_{\mathbf{R}}(p-1) x \varphi^{\prime} f(\varphi) d x \\
& =(1-p) \int_{\mathbf{R}} F(\varphi) d x \\
& =\frac{p-1}{p+1} K(\varphi) .
\end{aligned}
$$


Next, we note that the identities

$$
\int_{\mathbf{R}} \beta \varphi_{x}^{2}-c \varphi^{2}+\gamma\left(D_{x}^{-1} \varphi\right)^{2} d x=K(\varphi)
$$

and

$$
\int_{\mathbf{R}} \frac{1}{2} \beta \varphi_{x}^{2}+\frac{1}{2} c \varphi^{2}-\frac{3}{2} \gamma\left(D_{x}^{-1} \varphi\right)^{2} d x=-\frac{1}{p+1} K(\varphi),
$$

follow by multiplying (4.32) by $\varphi$ and $x \varphi^{\prime}$, respectively, and integrating. Combining these yields

$$
\int_{\mathbf{R}} c \varphi^{2} d x-2 \gamma \int_{\mathbf{R}}\left(D_{x}^{-1} \varphi\right)^{2} d x+\frac{p+3}{2(p+1)} K(\varphi)=0 .
$$

Next, we observe that

$$
\begin{aligned}
L^{\prime \prime}(\varphi)\left(x \varphi^{\prime}\right) & =-\beta\left(x \varphi^{\prime}\right)^{\prime \prime}-\gamma D_{x}^{-2}\left(x \varphi^{\prime}\right)-c x \varphi^{\prime}+f^{\prime}(\varphi) x \varphi^{\prime} \\
& =-\beta\left(x \varphi^{\prime \prime \prime}+2 \varphi^{\prime \prime}\right)-\gamma x D_{x}^{-1} \varphi+2 \gamma D_{x}^{-2} \varphi-c x \varphi^{\prime}+x f^{\prime}(\varphi) \varphi^{\prime} \\
& =-x\left(\beta \varphi^{\prime \prime \prime}+c \varphi^{\prime}+\gamma D_{x}^{-1} \varphi-f^{\prime}(\varphi) \varphi^{\prime}\right)-2 \beta \varphi^{\prime \prime}+2 \gamma D_{x}^{-2} \varphi
\end{aligned}
$$

Using equations (4.32) and (4.33), this simplifies to

$$
L^{\prime \prime}(\varphi)\left(x \varphi^{\prime}\right)=2 c \varphi+4 \gamma D_{x}^{-2} \varphi-2 f(\varphi)
$$

so

$$
\begin{aligned}
\left\langle L^{\prime \prime}(\varphi)\left(x \varphi^{\prime}\right), x \varphi^{\prime}\right\rangle & =\int_{\mathbf{R}}\left(2 c \varphi+4 \gamma D_{x}^{-2} \varphi-2 f(\varphi)\right) x \varphi^{\prime} d x \\
& =\int_{\mathbf{R}}-c \varphi^{2}+2 F(\varphi)+6 \gamma\left(D_{x}^{-1} \varphi\right)^{2} d x .
\end{aligned}
$$

Together with (4.38), this implies

$$
\begin{aligned}
\left\langle L^{\prime \prime}(\varphi)\left(x \varphi^{\prime}\right), x \varphi^{\prime}\right\rangle & =4 \gamma \int_{\mathbf{R}}\left(D_{x}^{-1} \varphi\right)^{2} d x+\left(\frac{p+3}{2(p+1)}-\frac{2}{p+1}\right) K(\varphi) \\
& =4 \gamma \int_{\mathbf{R}}\left(D_{x}^{-1} \varphi\right)^{2} d x+\frac{p-1}{2(p+1)} K(\varphi)
\end{aligned}
$$

as claimed. Therefore we deduce from (4.29), (4.34) and (4.35) that

$$
\begin{aligned}
& \left\langle L^{\prime \prime}(\varphi) \phi^{\prime}, \phi^{\prime}\right\rangle=\left\langle L^{\prime \prime}(\varphi) \varphi, \varphi\right\rangle+4\left\langle L^{\prime \prime}(\varphi) \varphi, x \varphi^{\prime}\right\rangle+4\left\langle L^{\prime \prime}(\varphi)\left(x \varphi^{\prime}\right), x \varphi^{\prime}\right\rangle \\
& =(1-p) K(\varphi)+\frac{4(p-1)}{p+1} K(\varphi)+\frac{2(p-1)}{p+1} K(\varphi)+16 \gamma \int_{\mathbf{R}}\left(D_{x}^{-1} \varphi\right)^{2} d x \\
& =\frac{(p-1)(5-p)}{p+1} K(\varphi)+16 \gamma \int_{\mathbf{R}}\left(D_{x}^{-1} \varphi\right)^{2} d x .
\end{aligned}
$$

This completes the proof of the Lemma.

Corollary 4.12. Let $\phi$ be defined in Lemma 4.11. Then $\left\langle L^{\prime \prime}(\varphi) \phi^{\prime}, \phi^{\prime}\right\rangle<0$, if 
(i) $c<0, p>5$, and $\gamma<\gamma_{0}$, for some small $\gamma_{0}>0$,

(ii) $c \leq 0$ and $p>5+4 \sqrt{2}$, and $\gamma>0$ or

(iii) $0<c<2 \sqrt{\gamma \beta}$ and $p>p_{0}$ with $p_{0}=\frac{10+k+\sqrt{(10+k)^{2}+4(7+k)}}{2}$ and $k=$ $8\left(\frac{2 \sqrt{\beta \gamma}}{2 \sqrt{\beta \gamma}-c}-1\right)>0$.

Proof. To prove (i), we first claim that

$$
\lim _{\gamma \rightarrow 0} \gamma \int_{\mathbf{R}}\left(D_{x}^{-1} \varphi\right)^{2} d x=0
$$

In fact, in view of (4.36), we have

$$
\gamma \int_{\mathbf{R}}\left(D_{x}^{-1} \varphi\right)^{2} d x=\int_{\mathbf{R}} c \varphi^{2}-\beta \varphi_{x}^{2} d x+K(\varphi)=\int_{\varphi} c \varphi^{2}-\beta \varphi_{x}^{2} d x+(m(\beta, c, \gamma))^{\frac{p+1}{p-1}}
$$

It is thereby inferred from Lemma 2.7 and Theorem 2.5 that

$$
\begin{aligned}
\lim _{\gamma \rightarrow 0} \gamma \int_{\mathbf{R}}\left(D_{x}^{-1} \varphi\right)^{2} d x & =\int_{\mathbf{R}} c \varphi_{0}^{2}-\beta\left(\partial_{x} \varphi_{0}\right)^{2} d x+(m(\beta, c, 0))^{\frac{p+1}{p-1}} \\
& =-I\left(\varphi_{0} ; \beta, c, 0\right)+(m(\beta, c, 0))^{\frac{p+1}{p-1}}=0,
\end{aligned}
$$

where $\varphi_{0}$ is the ground-state solution of $(\mathrm{KdV})$ with $c<0$ This in turn implies that,

$$
\lim _{\gamma \rightarrow 0}\left\langle L^{\prime \prime}(\varphi) \phi^{\prime}, \phi^{\prime}\right\rangle=\frac{(p-1)(5-p)}{p+1}(m(\beta, c, 0))^{\frac{p+1}{p-1}}<0
$$

for $c<0$ and $p>5$. This proves (i). To prove (ii) and (iii), we use (4.38) to write

$$
\begin{aligned}
2 \gamma \int_{\mathbf{R}}\left(D_{x}^{-1} \varphi\right)^{2} d x & =\frac{p+3}{2(p+1)} K(\varphi)+c \int_{\mathbf{R}} \varphi^{2} d x \\
& =\frac{p+3}{2(p+1)} K(\varphi)-K(\varphi)+\int_{\mathbf{R}} \beta \varphi_{x}^{2}+\gamma\left(D_{x}^{-1} \varphi\right)^{2} d x \\
& \leq\left(\frac{p+3}{2(p+1)}-1+\max \left\{1, \frac{2 \sqrt{\beta \gamma}}{2 \sqrt{\beta \gamma}-c}\right\}\right) K(\varphi) .
\end{aligned}
$$

Therefore it follows from formula (ii) in Lemma 4.11 that

$$
\left\langle L^{\prime \prime}(\varphi) \phi^{\prime}, \phi^{\prime}\right\rangle \leq\left(\frac{(p-1)(5-p)}{p+1}+\frac{4(p+3)}{p+1}-8(1-\rho)\right) K(\varphi)
$$

where $\rho=\max \left\{1, \frac{2 \sqrt{\beta \gamma}}{2 \sqrt{\beta \gamma}-c}\right\}$. If $c \leq 0$, then $\rho=1$ and it follows that

$$
\begin{aligned}
\left\langle L^{\prime \prime}(\varphi) \phi^{\prime}, \phi^{\prime}\right\rangle & \leq\left(\frac{(p-1)(5-p)}{p+1}+\frac{4(p+3)}{p+1}\right) K(\varphi) \\
& =-\frac{1}{p+1}(p-(5-4 \sqrt{2}))(p-(5+4 \sqrt{2})) K(\varphi)<0
\end{aligned}
$$


under the assumption of $p$ in (ii). If the assumption (iii) is satisfied, then

$$
p^{2}-(10+k) p-(7+k)>0
$$

where $k=8(\rho-1)>0$. It thus follows from (4.35) that

$$
\left\langle L^{\prime \prime}(\varphi) \phi^{\prime}, \phi^{\prime}\right\rangle=-\frac{1}{p+1}\left(p^{2}-(10+k) p-(7+k)\right) K(\varphi)<0 .
$$

The proof of the Corollary is complete.

\section{$5 \quad$ Numerical Results}

We now present some numerical computations of $d(c)$ for the nonlinearity $f(u)=(-u)^{p}$ for $p \geq 3$ an integer. The case $p=2$ is equation (1.1), and was considered in [15]. The strategy for computing $d$ is to first compute numerically the solutions of the solitary wave equation (2.1) using a shooting method. Then, using equation (3.3), we compute $d(c)$ and use a difference quotient to approximate $d^{\prime \prime}(c)$. The scaling property of $d$ in Lemma 3.2 helps to reduce the calculations from the domain $c<2 \sqrt{\beta \gamma}$ to two finite line segments. To see this, substitute $r=1 / 2 \sqrt{\beta \gamma}$ and $s=(4 \gamma / \beta)^{1 / 4}$ in the relation in Lemma 3.2 to get

$$
d(\beta, c, \gamma)=\beta^{\frac{p+1}{2(p-1)}+\frac{1}{4}}(4 \gamma)^{\frac{p+1}{2(p-1)}-\frac{1}{4}} d(1, c / 2 \sqrt{\beta \gamma}, 1 / 4) .
$$

Thus the value of $d$ along any surface of the form

$$
S_{\alpha}=\{(\beta, c, \gamma) \mid c / 2 \sqrt{\beta \gamma}=\alpha\}
$$

is determined by its value at any single point on that surface. We therefore need only compute $d$ along some set of paths which crosses every such surface. We make the following choice. Let $\Gamma_{1}=\{(1, c, 1 / 4) \mid-1 \leq c<1\}$ and $\Gamma_{2}=\{(1,-1, \gamma) \mid 0<\gamma \leq 1 / 4\}$. Then $\Gamma_{1}$ crosses $S_{\alpha}$ for $-1 \leq \alpha<1$, and $\Gamma_{2}$ crosses $S_{\alpha}$ for $\alpha \leq-1$. The paths $\Gamma_{1}$ and $\Gamma_{2}$ are shown below in the plane $\beta=1$.

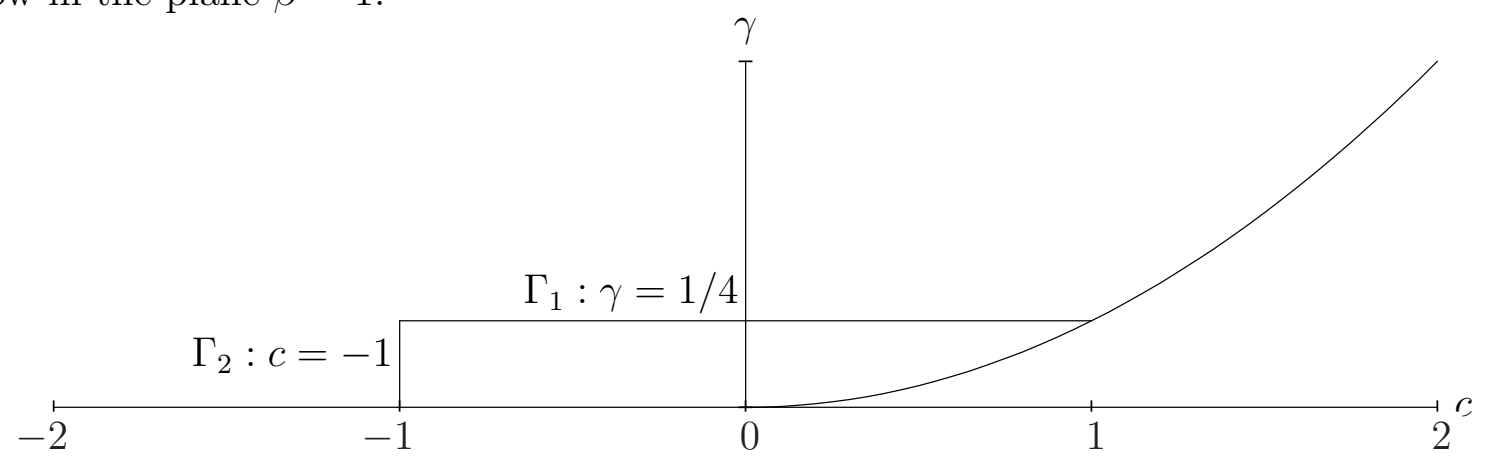

We now consider the sign of $d_{c c}$ along these curves.

Along $\Gamma_{1}$. Differentiating (5.1) twice with respect to $c$ gives

$$
d_{c c}(\beta, c, \gamma)=\beta^{\frac{p+1}{2(p-1)}-\frac{3}{4}}(4 \gamma)^{\frac{p+1}{2(p-1)}-\frac{5}{4}} d_{c c}(1, c / 2 \sqrt{\beta \gamma}, 1 / 4)
$$


Since $\beta>0$ and $\gamma>0$, it follows that the sign of $d_{c c}$ within $S_{\alpha}$ is determined by the sign of $d_{c c}(1, \alpha, 1 / 4)$.

Along $\Gamma_{2}$. Using Lemma 3.2 again, we deduce that

$$
d(1, c, \gamma)=(-c)^{\frac{p+3}{2(p-1)}} d\left(1,-1, \gamma / c^{2}\right)
$$

for $c<0$, so setting $q=\frac{p+3}{2(p-1)}$ and differentiating with respect to $c$ gives

$$
\begin{aligned}
d_{c}(1, c, \gamma) & =-q(-c)^{q-1} d\left(1,-1, \gamma / c^{2}\right)-\frac{2 \gamma}{c^{3}}(-c)^{q} d_{\gamma}\left(1,-1, \gamma / c^{2}\right) \\
& =-q(-c)^{q-1} d\left(1,-1, \gamma / c^{2}\right)+2 \gamma(-c)^{q-3} d_{\gamma}\left(1,-1, \gamma / c^{2}\right)
\end{aligned}
$$

and

$$
\begin{aligned}
d_{c c}(1, c, \gamma)= & q(q-1)(-c)^{q-2} d\left(1,-1, \gamma / c^{2}\right)+\frac{2 q \gamma}{c^{3}}(-c)^{q-1} d_{\gamma}\left(1,-1, \gamma / c^{2}\right) \\
& -2 \gamma(q-3)(-c)^{q-4} d_{\gamma}\left(1,-1, \gamma / c^{2}\right)-\frac{4 \gamma^{2}}{c^{3}}(-c)^{q-3} d_{\gamma \gamma}\left(1,-1, \gamma / c^{2}\right) \\
= & q(q-1)(-c)^{q-2} d\left(1,-1, \gamma / c^{2}\right)-2 \gamma(2 q-3)(-c)^{q-4} d_{\gamma}\left(1,-1, \gamma / c^{2}\right) \\
& +4 \gamma^{2}(-c)^{q-6} d_{\gamma \gamma}\left(1,-1, \gamma / c^{2}\right) .
\end{aligned}
$$

Setting $r=\gamma / c^{2}$, this simplifies to $d_{c c}(1, c, \gamma)=(-c)^{q-2} g(r)$, where

$$
g(r)=4 r^{2} d_{\gamma \gamma}(1,-1, r)-2(2 q-3) r d_{\gamma}(1,-1, r)+q(q-1) d(1,-1, r) .
$$

So it suffices to determine the sign of $g(r)$ for $0<r \leq \frac{1}{4}$.

Below we consider the nonlinearity $f(u)=(-u)^{p}$ for several values of $p$. We remark that the case $p=2$ is the classical Ostrovsky equation (1.1), for which it was shown by the authors in [15] using the same numerical method that $d^{\prime \prime}(c)$ is positive for all $\beta, \gamma$ and $c<2 \sqrt{\beta \gamma}$.
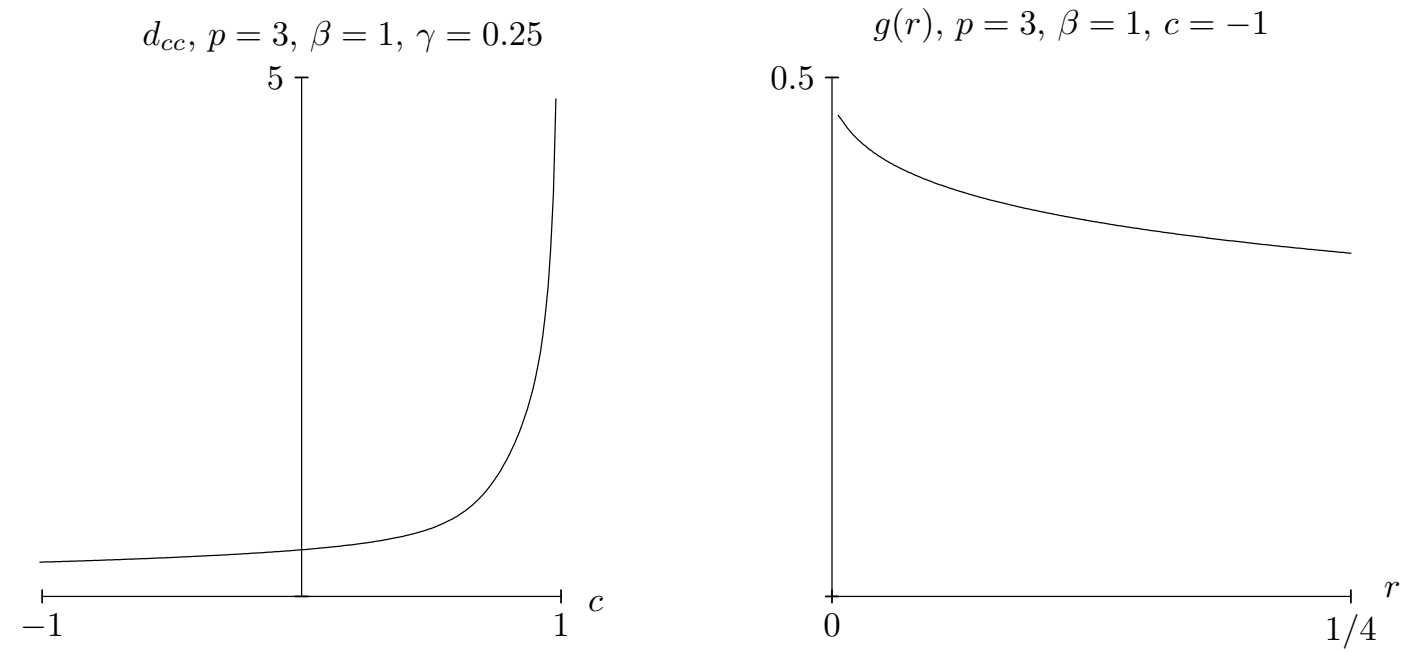


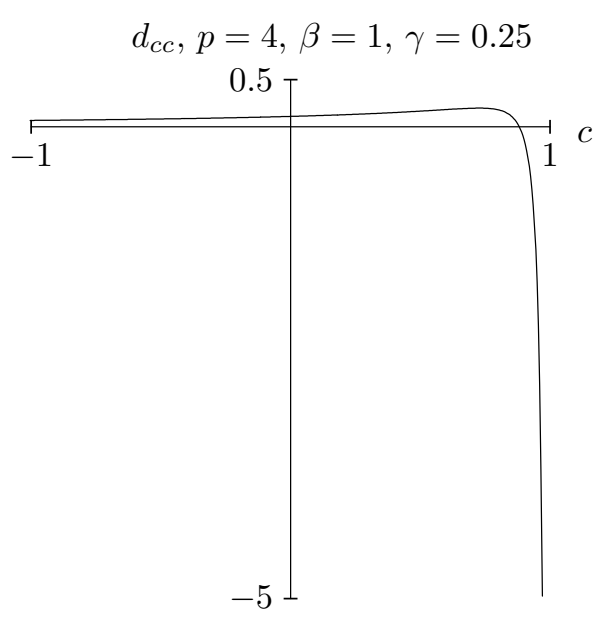

$g(r), p=4, \beta=1, c=-1$
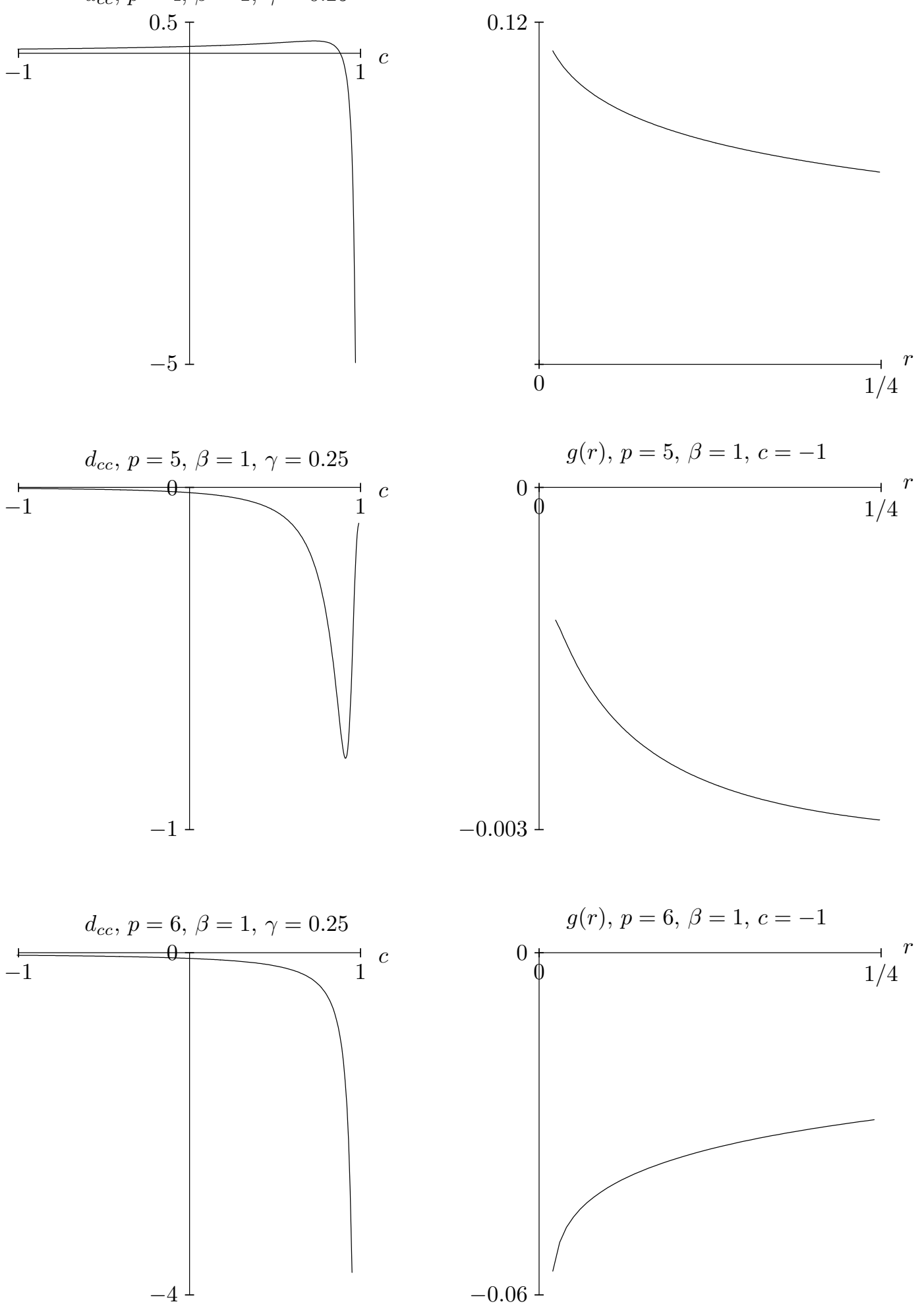

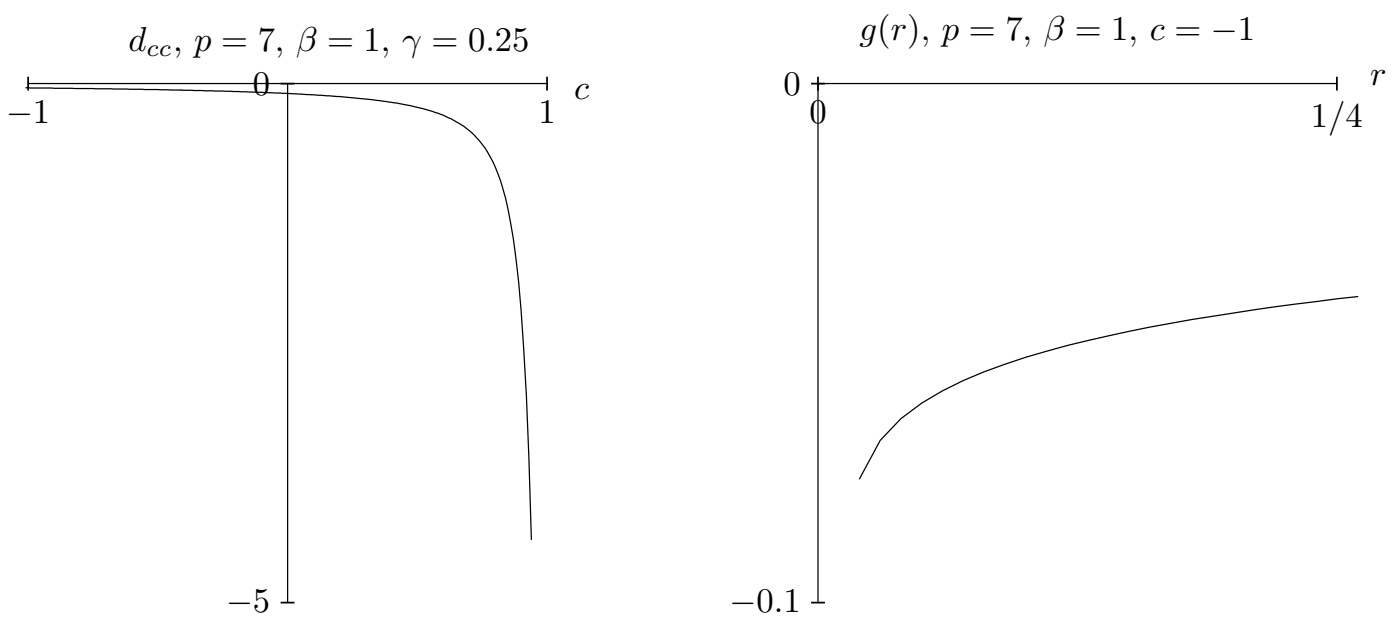

Using Theorems (3.1) and (4.3), we arrive at the following conclusions.

- When $p=3$, all solitary waves are stable for $c<2 \sqrt{\beta \gamma}$.

- When $p=4$ there exists $\alpha_{0}(\approx 0.88)$ such that solitary waves are stable for $\frac{c}{2 \sqrt{\beta \gamma}}<\alpha_{0}$ and solitary waves are unstable for $\alpha_{0}<\frac{c}{2 \sqrt{\beta \gamma}}<1$.

- When $p=5,6$ or 7 , all solitary waves are unstable for $c<2 \sqrt{\beta \gamma}$.

The case $p=4$ seems most interesting due to the change of stability. We conjecture that for all $p \geq 5$, solitary waves are unstable.

\section{Acknowledgement}

The authors are grateful for the constructive suggestions made by the referees.

\section{References}

[1] Angulo, J., On the instability of solitary waves solutions of the generalized Benjamin equation. Adv. Differential Equations 8 (2003), 55-82

[2] Benilov, E. S., On the surface waves in a shallow channel with an uneven bottom, Stud. Appl. Math., 87(1992), 1-14.

[3] Bona, J. And LiU, Yue, Instability of solitary wave solutions of the 3-dimensional Kadomtsev-Petviashvili equation, Adv. Differential Equations, 7(2002), 1-23.

[4] De Boundo, A. And Saut, J. C., Solitary waves of generalized KadomtsevPetviashvili equations, Ann. Inst. H. Poincaré Anal. Non Lin'eaire, A 14(1997), 211236.

[5] Bona, J., Souganidis, P. And Strauss, W., Stability and instability of solitary waves of Korteweg-de Vries type, Proc. Royal Soc. London Ser. A 411(1987), 395-412.

[6] Fr'ohlich, J., Lieb, E. H., And Loss, M., Stability of coulomb systems with magnetic fields I. The one electron atom, Commun. Math. Phys., 104(1986), 251-270. 
[7] Galkin, V. N. And Stepanyants, Y. A., On the existence of stationary solitary waves in a rotating field, J. Appl. Math. Mech., 55 (1991), 939-943.

[8] Gilman, O. A., Grimshaw, R., and Stepanyants, Y. A., Approximate and numerical solutions of the stationary Ostrovsky equation, Stud. Appl. Math., 95(1995), 115-126.

[9] Grimshaw, R., Evolution equations for weakly nonlinear long internal waves in a rotating fluid, Stud. Appl. Math., 73(1985), 1-33.

[10] Goncalves Ribeiro, J., Instability of symmetric stationary states for some nonlinear Schrödinger equations with an external magnetic field, Ann. Inst. H. Poincaré, Phys. Théore. , 54(1992), 109-145, 403-433.

[11] Guo, Y. And Rein, G., Existence and stability of Camm type steady states in galactic dynamics, Indiana U. Math. J., 48(1999), 1237-1255.

[12] Kadomtsev, B. B. And Petviashvili, V. I., On the stability of solitary waves in weakly dispersive media, Sov. Phys. Dokl., 15(6)(1970), 593-541.

[13] LeOnov, A., The effect of earth rotation on the propagation of weak nonlinear surface and internal long oceanic waves, Annals New York Acad. Sci., 373 (1981), 150-159.

[14] Levandosky, S., A stability analysis of fifth-order water wave models, Physica D, 125 (1999), 222-240.

[15] Levandosky, S. And Liu, Yue, Stability and weak rotation limit of solitary waves of the Ostrovsky equation, preprint.

[16] Lions, P. L., The concentration compactness principle in the calculus of variations. The locally compact case, Part 1 and Part 2, Ann. Inst. H. Poincaré, Anal. Nonlinéaire, 1(1984), 109-145, 223-283.

[17] LiU, YUE, Stability of solitary waves for the Ostrovsky equation with weak rotation, submitted.

[18] Liu, Yue and Varlamov, V., Stability of solitary waves and weak rotation limit for the Ostrovsky equation, J. Diff. Eqns., 203(2004), 159-183.

[19] Miura, R. M., Gardner, C. S., And Kruskal, M. D., Korteweg-de Vries equation and generalizations II, Existence conservation laws and constant of motion, J. Math. Phys., 9(1968), 1204-1209.

[20] Ostrovsky, L. A., Nonlinear internal waves in a rotating ocean, Okeanologia, 18(1978), 181-191.

[21] Ostrovaky, L. A. and Stepanyants, Y. A., Nonlinear surface and internal waves in rotating fluids, Research Reports in Physics, Nonlinear Waves 3, Springer, Berlin, Heidelberg, 1990. 
[22] ShrirA, V., Propagation of long non-liear waves in a layer of a rotating fluid, Iza. Akad. Nauk SSSR, Fiz. Atmosfery i Okeana, 17 (1981), 76-81.

[23] Shrira, V., On long essentially non-linear waves in a rotating ocean, Iza. Akad. Nauk SSSR, Fiz. Atmosfery i Okeana, 22 (1986), 395-405.

[24] Shatah, J. Stable standing waves of nonlinear Klein-Gordon equations, Comm. Math. Phys., 91(1983), 313-327.

[25] Shatah, J. And Strauss, W., Instability of nonlinear bound states, Comm. Math. Phys., 100(1985), 173-190.

[26] Varlamov, V. And Liu, Yue, Cauchy problem for the Ostrovsky equation, Discrete Dynam. Systems, 10(2004), 731-751. 